\title{
Calibration and comparison of VIV wake oscillator models for low mass ratio structures
}

Victoria Kurushina $^{1,2}$, Ekaterina Pavlovskaia ${ }^{1}$, Andrey Postnikov ${ }^{3}$, Marian Wiercigroch $^{1}$

${ }^{1}$ Centre for Applied Dynamics Research, School of Engineering, University of Aberdeen, Kings'College, Aberdeen, Scotland, United Kingdom, AB24 3FX

${ }^{2}$ Department of Transport of Hydrocarbon Resources, Institute of Transport, Industrial University of Tyumen, 38 Volodarskogo Street, Tyumen, Russia, 625040

${ }^{3}$ School of Engineering, University of Lincoln, Brayford Pool, Lincoln, United Kingdom, LN6 7TS

\section{Abstract}

In this work, Vortex-Induced Vibration (VIV) of a rigid cylinder on elastic supports is reviewed and modelled. We consider a cylinder moving both cross-flow and in-line subjected to fluid forces calculated as proposed in [1]. A suite of wake oscillator models is investigated with different types of nonlinear damping in the fluid equation, where sets of model coefficients are optimized for each variation of the nonlinear damping using the constrained nonlinear minimization. The calibration is based on the experimental data presented by Stappenbelt and Lalji [2]. The largest displacement amplitude is used as the main calibration criterion to determine empirical coefficients of the fluid equations. The frequency difference between the beginning of resonance evaluated from the base model [1] and the experimental data [2] is used as an additional coefficient for calibration.

The developed models are cross-checked using four sources of published experimental data [3-6]. This allows to identify the most suitable wake oscillator models which are applicable for a wide variety of experimental data. The main outcome of this research is a library of calibrated models describing fluid-structure interactions at low mass ratio. Specifically, it was shown that the Rayleigh damping is the most suitable for cross-flow equation, whereas the Van der Pol damping is preferable for in-line equation for these low mass ratio cases.

Keywords: Vortex-Induced Vibration, wake oscillator model, elastically mounted cylinder, low mass ratio, fluid nonlinearities, model calibration, validation

\section{Introduction}

Onshore and offshore structures are subject of strict safety regulations, and acceptable design implies requirements for accurate models of potentially dangerous phenomena. Slender structures are present almost everywhere in the form of tall buildings and skyscrapers, cranes, antennas, power lines, suspension bridges, umbilicals, risers and free spans of pipelines which deliver water, oil and gas. The deeper in the water and higher in the sky these structures are, more likely they can experience the interactions with the surrounding flows of water or air.

Vortex-Induced Vibration (VIV) develops when a slender structure interacts with a fluid flow. Vortices grow in the disturbed boundary layer and spread behind the structure, carried by the flow as a trace or travelling reflection of the structure left behind. Structure experiences additional loading due to pressure variation caused by the excited vortices, especially, when the frequency of vortex shedding gets closer to the natural frequency of the structure. In this case, the forces acting on the body increase significantly, and if the object is capable of moving around initial position, the increase in oscillation amplitudes is observed.

The current work is aimed to bring the recently developed wake oscillator model [1] closer to the design practice of VIV prediction. In modelling of VIV, a fluid oscillator was initially proposed almost as a "black box", where behaviour of the fluid is modelled with equation of "hidden flow variable" [7] with rather undefined physical nature. This approach has significantly progressed in the recent years and at the present moment, is developed for two-dimensional (2D) [1] or three-dimensional (3D) vibration [8, 9]. 
Large number of variables and parameters involved in analytical representations of VIV have been used in early works in this field, for example, by Roshko [10]. An important step on the way to simplify the problem was made in 1964 by Bishop and Hassan [11] who through experimental studies proposed an idea for the fluid equation. Few years after, Hartlen and Currie in 1970 [12] opened an era of creating various mathematical models of a fluid oscillator. This pioneering work employed the Rayleigh equation, with a lift coefficient as a variable used to estimate the fluid lift force acting on the structure.

From the earlier studies, it is clear that the wake oscillator approach controls the quality of VIV prediction by varying nonlinear damping terms. This idea was explored in several investigations, and it is worth to note the work by Landl [13]. He attempted to reconstruct the hysteresis effect observed in experimental data for transversal vibration using the new model. Consideration of the hysteresis effect is especially important for cases with mass ratio less than 6 [3], where the super-upper branch was observed. Landl [13] introduced the 5th order damping term into the fluid equation and mentioned that the model should be verified with a wider range of experimental data than was possible in 1975. For the available data, this 5th order term allowed to repeat the hysteresis effect, but did not allow to model the smaller vibration amplitudes correctly. In general, the work by Landl [13] illustrated how flexible the fluid oscillator could be to capture the development of the resonance state in terms of vibration amplitudes. Search for the best description of the hysteresis effect is not a part of the current research, but it can be considered as a logical continuation of exploring impact of the fluid nonlinearities in the future.

Another notable modification of wake oscillator appeared in 1999 when Krenk and Nielsen [14] proposed the combined nonlinear damping from Rayleigh and Van der Pol equations for transversal oscillations. The similar principle of modifying fluid equation was realized by Kim and Perkins in 2001 [15] for the two degrees-of-freedom system. They suggested coupling of two fluid oscillators with a number of positive quadratic damping terms.

In 2003, Nayfeh et al. [16] substantiated the application of a single wake oscillator to describe two degreesof-freedom (2DOFs) system where the structure is moving in both in-line and transversal directions to the flow. Three ways of connecting lift and drag were investigated. It was concluded that drag coefficient can be obtained as a dependence on the lift coefficient and the first derivative of the lift coefficient. The idea of this single oscillator [16] was recently implemented by Barbosa et al. [17] in the model for two degrees-of-freedom cylinder at various distances from a plane boundary. Single Van der Pol oscillator was employed in this work to represent fluctuations of the fluid force, and acceleration coupling was designed to provide the link with equations for both in-line and cross-flow motion of the structure.

Facchinetti et al. [18] in 2004 investigated different ways of coupling wake oscillator of Van der Pol type with the structural equation for one degree-of-freedom system, and they found that acceleration coupling provides a better quality of the fit than velocity or displacement couplings.

In 2008, applicability of Rayleigh and Van der Pol oscillators was discussed by Ogink and Metrikine [19]. One of the main suggestions made was an improved damping term for Van der Pol equation as $+\varepsilon \Omega_{R}\left(1-\frac{1}{a+b q^{2}}\right) \dot{q}$, with calibrated empirical constants $a$ and $b$, which was meant to predict more accurately oscillations of the lift force.

One of the latest studies by Bai and Qin [9] explores the ways to cover 2D and 3D VIV with models based on wake oscillators and the discrete point vortices. One part of their research is devoted to 2DOFs rigid structure, and the other part is focused on a 3D flexible riser. They choose the Rayleigh oscillator to describe the fluid forces with the reference to the very first model by Hartlen and Currie [12], and obtained a relatively good agreement with the experimental data by Jauvtis and Williamson [3]. The 3D model of a flexible structure described in [9] contains one harmonic and two nonlinear oscillators to describe fluid forces. 
Overall, most of the previously proposed models, including Iwan and Blevins [7], Nayfeh et al. [16], Facchinetti et al. [18], Ge et al. [20], Ogink and Metrikine [21], Bai and Qin [9], Pavlovskaia et al. [22], Postnikov et al. [1], Zanganeh and Srinil [8], Barbosa et al. [17] utilise classic Rayleigh or Van der Pol oscillators. Analysis of the previous wake oscillator studies leads to the conclusion that performance of these models can be markedly improved by simple modifications of the damping terms, but the question which damping terms are the most beneficial for accuracy of prediction in certain cases is not yet fully answered.

It is important to investigate the quality of the model prediction first for the rigid object moving in the flow. This would allow to build foundations for accurate modelling of a flexible structure which behaviour is more complex as a result of multi-frequency responses. The cases with one degree-of-freedom have been the research focus of different groups for a few decades, but the connection between predicted responses in two directions can be still improved. This is why, the scope of this paper is limited to a two degrees-of-freedom elastically-supported rigid cylinder.

Development of mathematical tools for VIV prediction is limited by the calibration requirements and, hence, the availability of experimental data for this phenomenon to apply for calibration becomes crucial. Rigid structures with two degrees-of-freedom were studied experimentally by Moe and Wu [23], Moe et al. [24], Gharib [25], Jeon and Gharib [26], Govardkhan and Williamson [27], Jauvtis and Williamson [3], Flemmimng and Williamson [28], Dahl et al. [4], Sanchis et al. [29], Blevins and Coughran [5], Kheirkhah et al. [30], Srinil et al. [6], Franzini et al. [31], Cagney and Balabani [32], Kim et al. [33], etc. The focus of the current paper is on prediction of the displacement amplitudes in in-line and cross-flow directions, and experimental data obtained by Stappenbelt and Lalji [2] for 2DOFs rigid structure for mass ratio of 2.36 are used for the models calibrations. The conducted work is focused on calibration and validation for low mass ratio from 2 to 4 , but the developed procedure can be applied for a different range of parameters as well.

The rest of the paper is organized as follows. Section 2 presents the set of fluid equations with various nonlinear damping which are considered during calibration. Section 3 reveals the system of priorities and rationale behind calculating an objective function to obtain the best fit for the resonance curve based on the chosen experimental results. Here, a frequency difference between the beginning of resonance according to mathematical model and according to experimental data [2] is identified as an additional coefficient for

calibration. Section 4 demonstrates the results of calibration with the in-line and cross-flow displacement records for mass ratio of 2.36 from the paper [2] and the validation using cases from different experimental set-ups where the super-upper branch is present. Section 5 summarises the performed study and identifies the directions for future research.

\section{Model and fluid nonlinearities}

In this work, free vibration of the elastically mounted rigid cylinder capable of oscillating in streamwise (in-line) and cross-flow directions are considered, and the structure (previously studied by Postnikov et al. [1]) is shown in Fig. 1. It is characterized by structural mass $m_{s}$, diameter $D$, structural damping $r_{s}$ and stiffness of elastic support $k$, in both in-line $(\bar{X})$ and cross-flow $(\bar{Y})$ direction.

In the mathematical description of this system, the following non-dimensional parameters are used:

$$
\omega_{n}=\sqrt{\frac{k}{m_{*}}} ; \quad \omega_{s t}=\frac{\omega_{n}}{\omega_{0}} ; \quad \zeta=\frac{r_{s}}{2 \omega_{0} m_{*}} ; \quad \mu=\frac{4 m_{s}}{\pi \rho_{f} D^{2}} ;
$$

where $\omega_{n}$ is natural frequency of the structure, $\omega_{0}$ is reference frequency, and $\rho_{f}$ is fluid density. Then $\zeta$ is dimensionless damping ratio, $\omega_{s t}$ is dimensionless natural frequency of the structure, and $\mu$ is mass ratio. Mass 
$m_{*}$ includes structural mass and fluid added mass, where $C_{A}$ is fluid added mass coefficient:

$$
m_{*}=\left(\mu+C_{A}\right) \frac{\pi \rho_{f} D^{2}}{4} .
$$

Time $t$, in-line displacement $x$ and cross-flow displacement $y$ of the centre of the structure are nondimensionalized as $\tau, X$ and $Y$ respectively:

$$
\tau=\omega_{0} t ; \quad X=\frac{x}{D} ; \quad Y=\frac{y}{D} .
$$

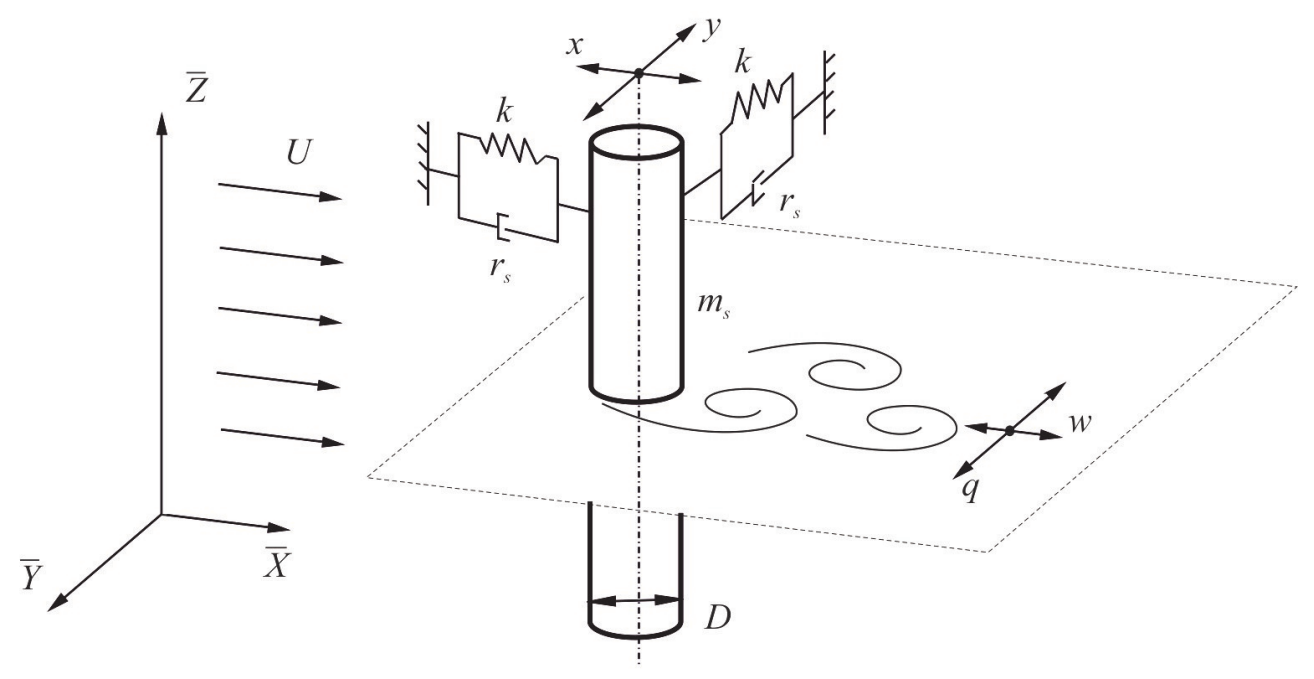

Figure 1: The model of two degrees-of-freedom elastically-supported rigid structure interacting with the uniform flow of velocity $U$.

The fluid flow is assumed to be uniform, and characterized by velocity $U$ and vortex shedding frequency $\Omega_{f}$, and, in the nondimensional form, reduced velocity $U_{R}$ and vortex shedding frequency $\Omega_{R}$ are used:

$$
U_{R}=\frac{2 \pi U}{\omega_{n} D} ; \quad \Omega_{R}=\frac{\Omega_{f}}{\omega_{0}}=\frac{2 \pi S t U}{\omega_{0} D} .
$$

Strouhal number $S t$ is assumed to be constant and equal to 0.2 in this study for all considered cases. This assumption is justified by the work of Blevins [34], where it is shown that the Strouhal number has a value of 0.2 for a wide range of Reynolds numbers, but also by limited availability of the information about the actual Strouhal number in the published experimental data. Also throughout this work, we use $\omega_{0}=\omega_{n}$, and hence, $\omega_{s t}=1$.

The lift and drag forces acting on the cylinder are calculated using fluctuating lift and $\operatorname{drag}$ coefficients $C_{L}$ and $C_{D}^{f l}$ which are represented as nondimensional in-line $w$ and cross-flow $q$ wake coefficients:

$$
w=\frac{2 C_{D}^{f l}}{C_{D 0}^{f l}} ; \quad q=\frac{2 C_{L}}{C_{L 0}} .
$$

Here, fluctuating drag $C_{D}^{f l}$ is defined as a difference of drag coefficient $C_{D}$ of a moving structure and drag coefficient $C_{D 0}$ of a stationary structure, $C_{D}^{f l}=C_{D}-C_{D 0} . C_{L 0}$ and $C_{D 0}^{f l}$ are lift and fluctuating drag coefficients of a stationary structure respectively.

In the current study, the model structure developed in [1] is used for describing the fluid forces acting on the cylinder. In this approach the acceleration coupling suggested by Facchinetti et al. [18] is used containing coupling coefficients $A_{x}$ and $A_{y}$ for in-line and cross-flow wake oscillator equations respectively, and frequency 
doubling effect is introduced in the in-line fluid equation. In [1], Van der Pol damping is employed in both in-line and cross-flow directions with damping terms in the form of $\varepsilon_{x} \Omega_{R}\left(w^{2}-1\right) \dot{w}$ and $\varepsilon_{y} \Omega_{R}\left(q^{2}-1\right) \dot{q}$. Thus, the equations of motion are [1]:

$$
\begin{aligned}
& \ddot{X}+2 \zeta \dot{X}+\omega_{s t}^{2} X=\frac{a}{2 \pi S t} \Omega_{R}^{2}+\frac{b}{4 \pi S t} \Omega_{R}^{2} w-2 a \Omega_{R} \dot{X}+\frac{c}{2} \Omega_{R} q \dot{Y}+a \pi S t \dot{Y} \dot{Y}+2 a \pi S t \dot{X} \dot{X}-b \Omega_{R} w \dot{X} \\
& \ddot{Y}+2 \zeta \dot{Y}+\omega_{s t}^{2} Y=\frac{c \Omega_{R}^{2}}{4 \pi S t} q-a \Omega_{R} \dot{Y}+2 a \pi S t \dot{X} \dot{Y}-\frac{b}{2} w \dot{Y} \Omega_{R}-c q \dot{X} \Omega_{R} ; \\
& \ddot{w}+2 \varepsilon_{x} \Omega_{R}\left(w^{2}-1\right) \dot{w}+4 \Omega_{R}^{2} w=A_{x} \ddot{X} \\
& \ddot{q}+\varepsilon_{y} \Omega_{R}\left(q^{2}-1\right) \dot{q}+\Omega_{R}^{2} q=A_{y} \ddot{Y}
\end{aligned}
$$

where

$$
a=\frac{C_{D 0} \rho_{f} D^{2}}{4 \pi m_{*} S t} ; \quad b=\frac{C_{D 0}^{f l} \rho_{f} D^{2}}{4 \pi m_{*} S t} ; \quad c=\frac{C_{L 0} \rho_{f} D^{2}}{4 \pi m_{*} S t} .
$$

As was discussed earlier, there are a few versions of damping in the fluid equation employed throughout the history of wake oscillator method, as proposed by Rayleigh, Van der Pol, Landl, Krenk and Nielsen. Current study is focused on investigating the effects of the damping nonlinearities on the system response, and in-line and cross-flow equations for the considered fluid nonlinearities are presented in Table 1. In addition to the classic Van der Pol and Rayleigh equations, it is suggested to investigate also Modified Van der Pol and Modified Rayleigh equations where damping coefficients $\varepsilon_{x}$ and $\varepsilon_{y}$ are split into separate coefficients for each damping term as $\varepsilon_{x 1}, \varepsilon_{x 2}, \varepsilon_{y 1}, \varepsilon_{y 2}$. Landl and Krenk-Nielsen equations contain also coefficients $\varepsilon_{x 3}$ and $\varepsilon_{y 3}$ to regulate contribution of the third damping term of wake oscillator for in-line and cross-flow direction respectively.

Table 1: Different models of wake oscillators.

\begin{tabular}{|c|c|c|}
\hline Oscillator & In-line equation & Cross-flow equation \\
\hline Van der Pol & $\ddot{w}-2 \varepsilon_{x} \Omega_{R} \dot{w}+2 \varepsilon_{x} \Omega_{R} \dot{w} w^{2}+4 \Omega_{R}^{2} w=A_{x} \ddot{X} ;$ & $\ddot{q}-\varepsilon_{y} \Omega_{R} \dot{q}+\varepsilon_{y} \Omega_{R} \dot{q} q^{2}+\Omega_{R}^{2} q=A_{y} \ddot{Y} ;$ \\
\hline $\begin{array}{c}\text { Modified } \\
\text { Van der Pol }\end{array}$ & $\ddot{w}-2 \varepsilon_{x 1} \Omega_{R} \dot{w}+2 \varepsilon_{x 2} \Omega_{R} \dot{w} w^{2}+4 \Omega_{R}^{2} w=A_{x} \ddot{X} ;$ & $\ddot{q}-\varepsilon_{y 1} \Omega_{R} \dot{q}+\varepsilon_{y 2} \Omega_{R} \dot{q} q^{2}+\Omega_{R}^{2} q=A_{y} \ddot{Y} ;$ \\
\hline Rayleigh & $\ddot{w}-2 \varepsilon_{x} \Omega_{R} \dot{w}+2 \frac{\varepsilon_{x}}{\Omega_{R}} \dot{w}^{3}+4 \Omega_{R}^{2} w=A_{x} \ddot{X} ;$ & $\ddot{q}-\varepsilon_{y} \Omega_{R} \dot{q}+\frac{\varepsilon_{y}}{\Omega_{R}} \dot{q}^{3}+\Omega_{R}^{2} q=A_{y} \ddot{Y} ;$ \\
\hline $\begin{array}{c}\text { Modified } \\
\text { Rayleigh }\end{array}$ & $\ddot{w}-2 \varepsilon_{x 1} \Omega_{R} \dot{w}+2 \frac{\varepsilon_{x 2}}{\Omega_{R}} \dot{w}^{3}+4 \Omega_{R}^{2} w=A_{x} \ddot{X} ;$ & $\ddot{q}-\varepsilon_{y 1} \Omega_{R} \dot{q}+\frac{\varepsilon_{y 2}}{\Omega_{R}} \dot{q}^{3}+\Omega_{R}^{2} q=A_{y} \ddot{Y} ;$ \\
\hline Landl & $\ddot{w}+2 \Omega_{R} \dot{w}\left(\varepsilon_{x 1}-\varepsilon_{x 2} w^{2}+\varepsilon_{x 3} w^{4}\right)+4 \Omega_{R}^{2} w=A_{x} \ddot{X} ;$ & $\ddot{q}+\Omega_{R} \dot{q}\left(\varepsilon_{y 1}-\varepsilon_{y 2} q^{2}+\varepsilon_{y 3} q^{4}\right)+\Omega_{R}^{2} q=A_{y} \ddot{Y} ;$ \\
\hline $\begin{array}{l}\text { Krenk- } \\
\text { Nielsen }\end{array}$ & $\ddot{w}-2 \varepsilon_{x 1} \Omega_{R} \dot{w}+2 \varepsilon_{x 2} \Omega_{R} \dot{w} w^{2}+\frac{\varepsilon_{x 3}}{\Omega_{R}} \dot{w}^{3}+4 \Omega_{R}^{2} w=A_{x} \ddot{X} ;$ & $\ddot{q}-\varepsilon_{y 1} \Omega_{R} \dot{q}+\varepsilon_{y 2} \Omega_{R} \dot{q} q^{2}+\frac{\varepsilon_{y 3}}{\Omega_{R} \dot{q}^{3}+\Omega_{R}^{2} q=A_{y} \ddot{Y} ;}$ \\
\hline
\end{tabular}

As can be seen from Table 1, we retain the acceleration coupling proposed by Facchinetti et al. [18] for all considered cases along with frequency doubling effect for all in-line equations in the same way as it is introduced in $[1]$.

Introducing two wake equations allows managing the quality of VIV prediction for each direction by varying nonlinear damping. Hence, in this study, fluid nonlinearities are applied in couples, for example, the option 
"Rayleigh - Van der Pol" means that Rayleigh damping is applied in in-line wake equation, and Van der Pol damping is applied in cross-flow wake equation. Overall, 28 combinations of oscillators were investigated in this work as shown in Table 2 .

Table 2: Investigated combinations of oscillators (" $\checkmark$ " denotes the combinations covered in this research, and "-" means the combination is not covered).

\begin{tabular}{|c|c|c|c|c|c|c|}
\hline In-line Cross-flow & Rayleigh & $\begin{array}{l}\text { Modified } \\
\text { Rayleigh }\end{array}$ & Van der Pol & $\begin{array}{c}\text { Modified } \\
\text { Van der Pol }\end{array}$ & Landl & Krenk-Nielsen \\
\hline Van der Pol & $\checkmark$ & - & $\checkmark$ & - & $\checkmark$ & $\checkmark$ \\
\hline Modified Van der Pol & - & $\checkmark$ & - & $\checkmark$ & $\checkmark$ & $\checkmark$ \\
\hline Rayleigh & $\checkmark$ & - & $\checkmark$ & - & $\checkmark$ & $\checkmark$ \\
\hline Modified Rayleigh & - & $\checkmark$ & - & $\checkmark$ & $\checkmark$ & $\checkmark$ \\
\hline Landl & $\checkmark$ & $\checkmark$ & $\checkmark$ & $\checkmark$ & $\checkmark$ & $\checkmark$ \\
\hline Krenk-Nielsen & $\checkmark$ & $\checkmark$ & $\checkmark$ & $\checkmark$ & $\checkmark$ & $\checkmark$ \\
\hline
\end{tabular}

\section{Calibration}

Due to their inherent empirical nature, all wake oscillator models require tuning and systematic validation with experimental data. Calibration of the empirical coefficients of the model is a search of the best fit between the modelled and experimental response. In this study, the efforts are concentrated on predicting displacements of the structure rather than the values of oscillating fluid forces. Calibration of working coefficients is, in fact, solution of an optimization problem, and, to simplify the calculations, the focus is placed solely on displacements of the structure at the resonance state, especially, on super-upper branch with top displacement amplitudes. However, it should be noted that a similar work can be done in the future for lift and drag coefficients.

Calibration of semi-empirical models like wake oscillator is not well covered in the previous studies. Most of papers on VIV do not specify how exactly the working set of coefficients was found, which leads to an assumption that for most of the existing models these coefficients were selected manually. One of the exceptions is the recent study by Xu et al., 2015 [35], where three empirical constants are calibrated using genetic algorithm. This work demonstrates that the present state of art of computational algorithms allows to conduct the optimization in a systematic way and for multiple empirical coefficients.

In the current study, each of the models developed in the previous section is calibrated using constrained nonlinear minimization from Matlab Optimization Toolbox. Equations of motion described in the Section 2 are integrated using Matlab ode45 solver to obtain the amplitudes of displacement for a range of the flow velocities. Identification of the working sets of coefficients is based on the empirical data from [2] for low mass ratio of 2.36 . In these experimental series [2], the rigid structure has diameter of $0.0554 \mathrm{~m}$, natural frequency in still water $1.711 \mathrm{~Hz}$, structural damping ratio 0.006, aspect ratio 8. The behaviour of the structure is investigated in the range of flow velocity $0.33-1.52 \mathrm{~m} / \mathrm{s}$, which corresponds to Reynolds number interval from around 18300 to 84200 and reduced velocity interval from 3.5 to 16.0 . The considered case of mass ratio of 2.36 corresponds to mass-damping ratios $\mu \zeta=0.014$ and $\left(\mu+C_{A}\right) \zeta=0.02$. Then the largest developed dimensionless amplitudes of displacement are 1.61 and 0.225 for cross-flow and in-line directions respectively.

To perform the optimization procedure, the initial values of calibrated coefficients, and the lower and upper bounds of the considered range should be specified, and the selected values are given in Table 3 . They are 
based on the previous validations with the experimental data reported in [1] and on the preliminary calibrations conducted in this study.

Table 3: Initial set of coefficients and its' optimization constraints.

\begin{tabular}{|l|c|c|c|c|}
\hline Parameter & Symbol & Initial value & Lower bound & Upper bound \\
\hline Initial lift coefficient & $C_{L 0}$ & 0.8085 & 0.01 & 10.0 \\
Initial drag coefficient & $C_{D 0}$ & 1.3996 & 0.01 & 10.0 \\
Initial fluctuating drag coefficient & $C_{D}^{f l}$ & 0.0101 & 0.01 & 5.0 \\
In-line fluid damping parameter & $\varepsilon_{x}$ & 0.5932 & 0.00001 & 3.0 \\
Cross-flow fluid damping parameter & $\varepsilon_{y}$ & 0.0138 & 0.00001 & 3.0 \\
Coupling coefficient in in-line direction & $A_{x}$ & 11.9552 & 1.0 & 40.0 \\
Coupling coefficient in cross-flow direction & $A_{y}$ & 4.3201 & 1.0 & 40.0 \\
Fluid added mass coefficient & $C_{A}$ & 1.2878 & 0.1 & 10.0 \\
Lock-in delay coefficient & $K$ & 0.5 & -3 & 4 \\
\hline
\end{tabular}

Here, the objective function for the optimization is formulated as a minimized difference between the model prediction, $Y_{m}$, and experimental data, $Y_{e x p}$. The model prediction, $Y_{m}$, can be assessed as a statistical function from the generated signal, for example, maximum value, root mean square or standard deviation built in Matlab. This study is focused on using the maximum of a signal and its standard deviation, and samples of these functions are displayed in comparison in Fig. 2 for in-line and cross-flow displacements generated by the model. In the work by Stappenbelt and Lalji [2], amplitudes of displacement $x / D$ and $y / D$ are computed from 10 highest peak-to-peak values of the recorded experimental signal. There are no published recommendations or agreed procedure regarding the choice of signal statistics for calibration of wake oscillator class of models at the present moment. Hence, calibration of maximum of the generated displacement amplitude with the experimental data [2] appears to be a reasonable approach. However, calibration of a statistical function like standard deviation or other, which is less than maximum of displacement, with experimental data [2] would enable additional safety margin, if the final prediction will be made based on maximum of the model signal. Therefore, both the maximum and standard deviation are implemented further as two kinds of applied optimization schemes.

(a)

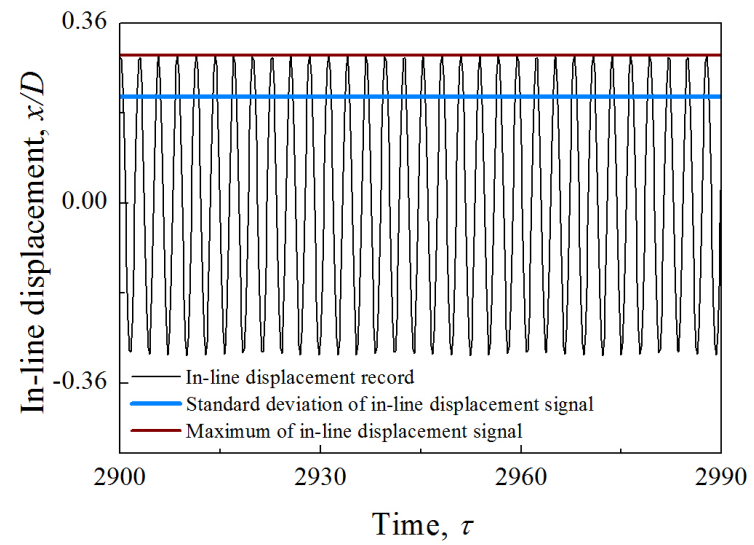

(b)

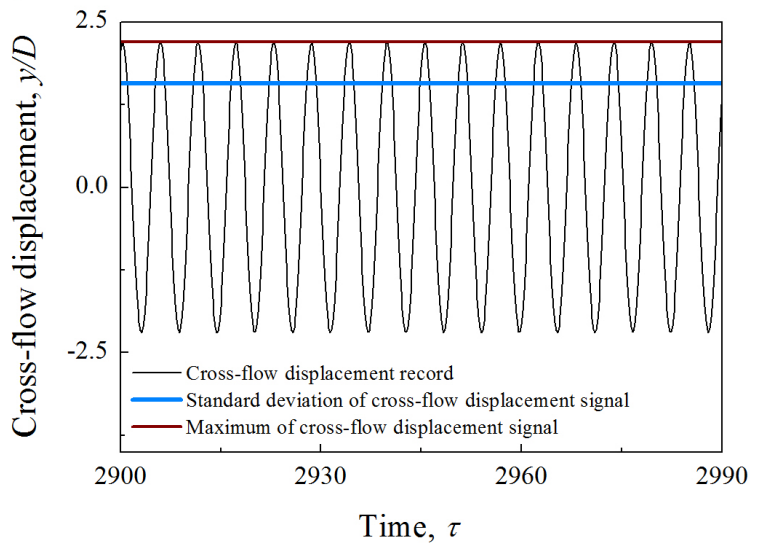

Figure 2: Standard deviation and maximum are illustrated here against displacement signals generated by the model: (a) for in-line oscillations; (b) for cross-flow oscillations.

In general, the optimization objective function for VIV problem should be designed taking into account the 
following aspects:

- The difference between the model and experiment should be assessed involving as few data points as possible to reduce the computational time for optimization procedure.

- The main priority of calibration is the correct prediction of the largest resonance amplitude and the nearby amplitudes to ensure the safe fit.

- The second priority is the lock-in start and the end of super-upper branch as the points where the dynamics of response changes.

Based on these considerations, the objective function is constructed using 11 out of 26 available experimental data points, and an example of the selected points at the resonance curve for mass ratio of 2.36 [2] is shown in Fig. 3. Thereby, the sum of differences between statistics from the response produced by the calibrated model $Y_{m}$ and experimental data $Y_{\exp }$ in the control point $i$ from [2] constitutes the minimized objective function. The authors suggest calculating the objective function as a sum of squared differences $S_{\text {sqrs }}$ or, alternatively, as a sum of relative errors $S_{r e l}$ :

$$
S_{s q r s}=\sum_{i=1}^{N} w_{i}\left(Y_{m i}-Y_{e x p_{i}}\right)^{2},
$$

or

$$
S_{r e l}=\sum_{i=1}^{N} w_{i} \frac{\left|Y_{m i}-Y_{e^{x p_{i}}}\right|}{Y_{\exp _{i}}}
$$

where $w_{i}$ is a weighting of the contribution of the error between the model data and experiment in the control point $i$ into the overall error, and $N=26$ is the number of available experimental points.

(a)

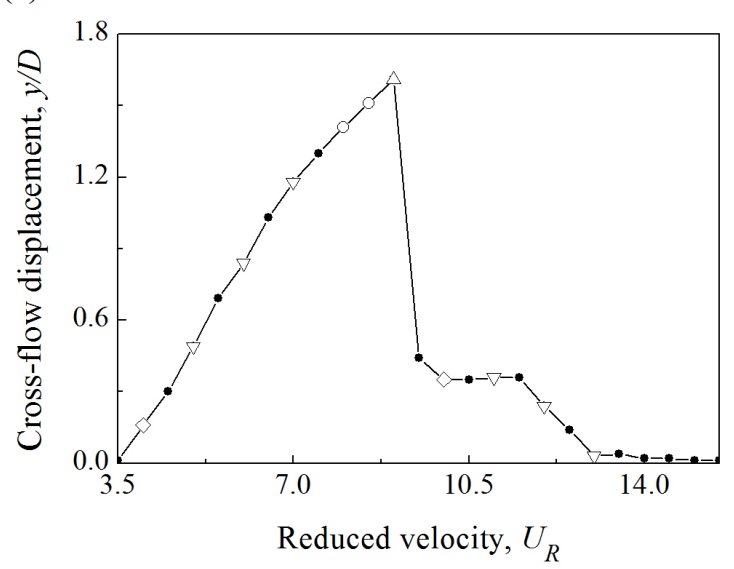

(b)

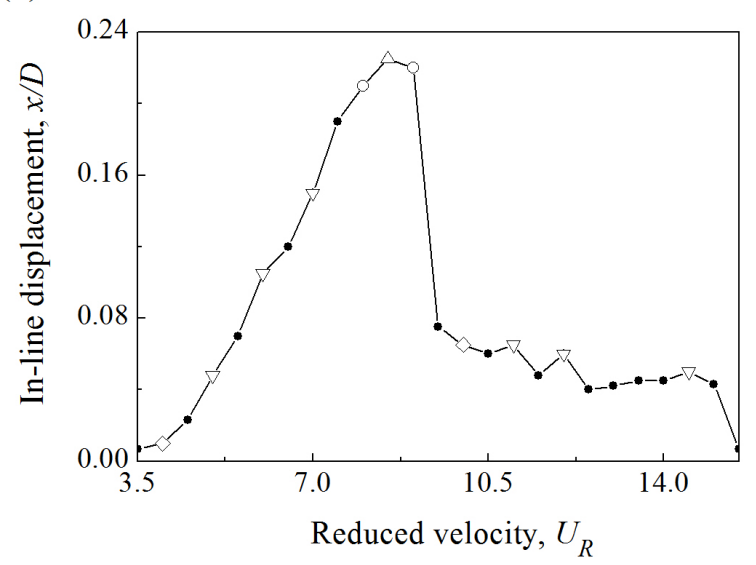

Figure 3: Dependence of the response amplitude on the reduced velocity obtained experimentally [2]. The selected data points on the resonance peaks are shown for (a) cross-flow and (b) in-line responses [2]: $\rightarrow$ are the values obtained in experiment [2] which are not used in the optimization procedure; $\triangle$ - the highest resonance value; $\bigcirc$ - the amplitudes near the highest resonance value; $\diamond$ - beginning of the resonance and end of the super-upper branch; $\nabla$ - intermediate points on the resonance peak.

A weighted contribution of the points into objective function is applied in order to maintain the quality of the super-upper branch prediction. Weighting of 4 is applied for the point with the highest value of displacement amplitude (see Fig. 3), weighting of 3 is applied for the points near the highest resonance value, and weighting 
of 2 emphasizes beginning of the resonance and end of the super-upper branch. Contribution of other points distributed along the resonance curve is weighted as 1 or 0 as shown in Table 4 . This system of priorities is aimed to reflect both the needs of scientists looking for the most accurate solutions, and the needs of industry to design offshore structures in respect to maximum expected excitation.

Table 4: Weighting coefficients used in the optimization procedure.

\begin{tabular}{|c|c|c|c|c|c|c|c|c|c|c|c|c|c|c|c|c|c|c|c|c|c|}
\hline \multicolumn{22}{|c|}{ Cross-flow displacement calibration } \\
\hline$i$ & 1 & 2 & 3 & 4 & 5 & 6 & 7 & 8 & 9 & 10 & 11 & 12 & 13 & 14 & 15 & 16 & 17 & 18 & 19 & 20 & $21-26$ \\
\hline$w_{i}$ & 0 & 2 & 0 & 1 & 0 & 1 & 0 & 1 & 0 & 3 & 3 & 4 & 0 & 2 & 0 & 1 & 0 & 1 & 0 & 1 & 0 \\
\hline \multicolumn{22}{|c|}{ In-line displacement calibration } \\
\hline$i$ & 1 & 2 & 3 & 4 & 5 & 6 & 7 & 8 & 9 & 10 & 11 & 12 & 13 & 14 & 15 & 16 & 17 & 18 & $19-22$ & 23 & $24-26$ \\
\hline$w_{i}$ & 0 & 2 & 0 & 1 & 0 & 1 & 0 & 1 & 0 & 3 & 4 & 3 & 0 & 2 & 0 & 1 & 0 & 1 & 0 & 1 & 0 \\
\hline
\end{tabular}

All the considered ways to compute an objective function for each modification of the model in this study are summarised in Table 5 as 8 optimization schemes. These schemes are distinguished by the way of calculating the amplitude of the displacement signal generated by the model, and by the target values - in-line or cross-flow displacement amplitudes [2]. For example, scheme "In-line 3", as described in Table 5, implies that the model variation is calibrated with respect to experimental in-line results from [2] by minimization of the weighted sum of absolute squared differences between experimental in-line response and standard deviation of the signal generated by the model in the control points.

Table 5: Optimization schemes to identify coefficients of the best fit.

\begin{tabular}{|l|l|l|l|}
\hline Scheme & Calibrated response & Statistics of signal & Identification of error \\
\hline In-line 1 & In-line displacement amplitude & Maximum & Absolute squared differences \\
\hline In-line 2 & In-line displacement amplitude & Maximum & Relative errors \\
\hline In-line 3 & In-line displacement amplitude & Standard deviation & Absolute squared differences \\
\hline In-line 4 & In-line displacement amplitude & Standard deviation & Relative errors \\
\hline Cross-flow 1 & Cross-flow displacement amplitude & Maximum & Absolute squared differences \\
\hline Cross-flow 2 & Cross-flow displacement amplitude & Maximum & Relative errors \\
\hline Cross-flow 3 & Cross-flow displacement amplitude & Standard deviation & Absolute squared differences \\
\hline Cross-flow 4 & Cross-flow displacement amplitude & Standard deviation & Relative errors \\
\hline
\end{tabular}

Preliminary calibrations using the algorithms described above revealed three important issues regarding calibration strategy for the considered model and, possibly, for the whole class of wake oscillator models. First of all, there was no leading scheme identified that would find the best set of coefficients for every model modification. Hence, it is essential to approach calibration with different strategies in parallel in order not to miss possible working sets. Secondly, the objective function has to be equipped with the "filter-out" system to exclude sets of coefficients, which:

- do not provide the desired quality in the highest value of the resonance;

- show significant deviation from initial and lower branches.

The applied optimization schemes can be refined further if more criteria of scientific or industrial nature are specified. 
Finally, the frequency difference between the beginning of the resonance according to the original model [1] and the experimental data was identified as an additional coefficient to be found during the calibration. The sample of this parallel horizontal transformation is given in Fig. 4. It can be interpreted as a delay between the ideal (theoretical) development of VIV at reduced velocity $U_{R m}$ and the reaction of structure in experimental conditions at reduced velocity $U_{\text {Rexp }}$. This difference can be measured and optimized and it is named here as the lock-in delay coefficient $K=U_{R e x p}-U_{R m}$. This lock-in delay to some extent may originate from the difference between actual Strouhal number for each experimental case and the unified value used in the current modelling as commented earlier. In most of cases, it was found that $K \in(0.5,1.5)$, which is reflected in Table 3. Introduction of this coefficient results in the following modification of the system of equations of motion:

(a)

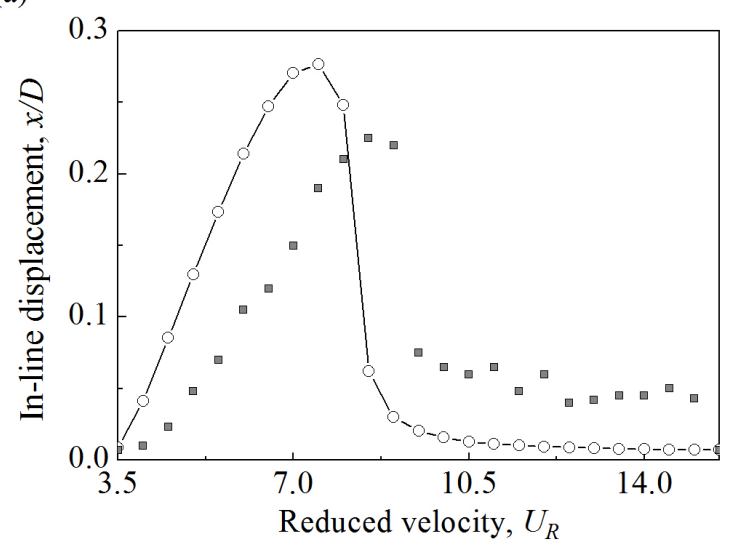

(c)

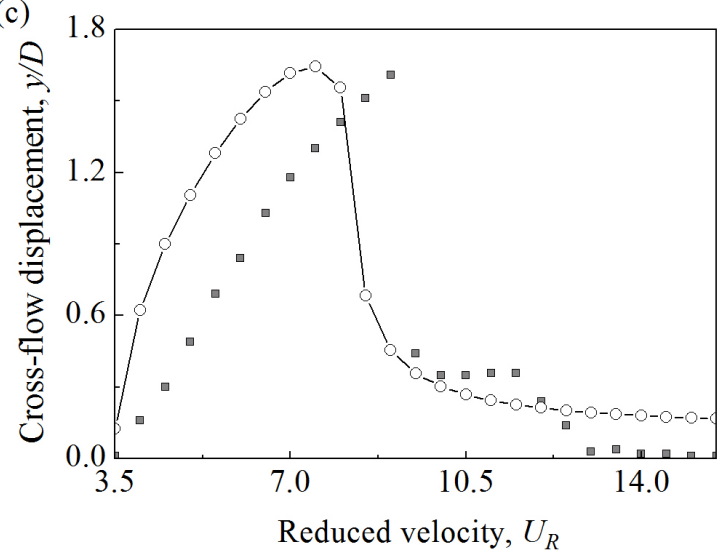

(b)

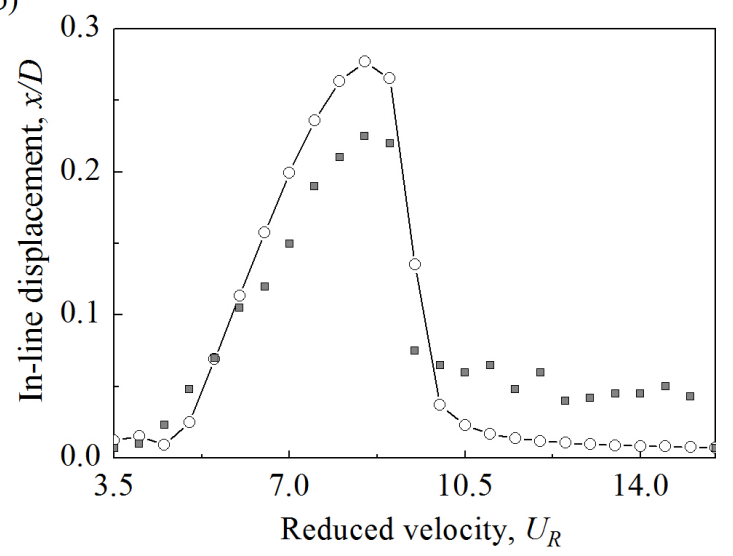

(d)

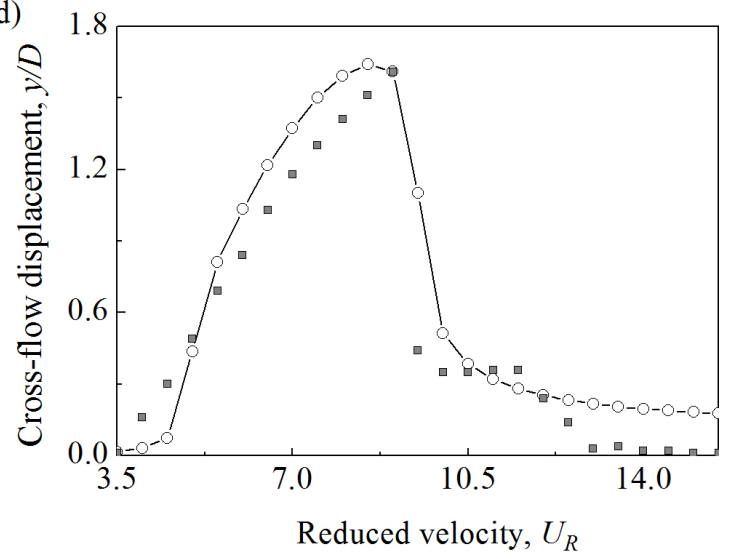

Figure 4: Displacement amplitudes obtained experimentally in comparison with predicted displacement amplitudes generated by one of the considered model versions over the range of reduced velocity of the flow: (a) in-line amplitudes before introducing delay coefficient $K$ in the model; (b) in-line amplitudes after introducing delay coefficient $K$; (c) cross-flow amplitudes before introducing delay coefficient $K$; (d) cross-flow amplitudes after introducing delay coefficient $K$. Calibrated lock-in delay for this model variation is $K=0.94 ;--$ is the model prediction obtained using standard deviation from the displacement signal generated by the model, and $\square$ denotes experimental measurements. 


$$
\begin{aligned}
\ddot{X} & +2 \zeta \dot{X}+\omega_{s t}^{2} X=\frac{a}{2 \pi S t}\left(\Omega_{R}-S t K\right)^{2}+\frac{b}{4 \pi S t}\left(\Omega_{R}-S t K\right)^{2} w-2 a\left(\Omega_{R}-S t K\right) \dot{X}+ \\
& +\frac{c}{2}\left(\Omega_{R}-S t K\right) q \dot{Y}+a \pi S t \dot{Y} \dot{Y}+2 a \pi S t \dot{X} \dot{X}-b\left(\Omega_{R}-S t K\right) w \dot{X} ; \\
\ddot{Y} & +2 \zeta \dot{Y}+\omega_{s t}^{2} Y=\frac{c\left(\Omega_{R}-S t K\right)^{2}}{4 \pi S t} q-a\left(\Omega_{R}-S t K\right) \dot{Y}+2 a \pi S t \dot{X} \dot{Y}- \\
& -\frac{b}{2} w \dot{Y}\left(\Omega_{R}-S t K\right)-c q \dot{X}\left(\Omega_{R}-S t K\right) ; \\
\ddot{w} & +2 \varepsilon_{x}\left(\Omega_{R}-S t K\right)\left(w^{2}-1\right) \dot{w}+4\left(\Omega_{R}-S t K\right)^{2} w=A_{x} \ddot{X} ; \\
\ddot{q} & +\varepsilon_{y}\left(\Omega_{R}-S t K\right)\left(q^{2}-1\right) \dot{q}+\left(\Omega_{R}-S t K\right)^{2} q=A_{y} \ddot{Y} ;
\end{aligned}
$$

Results of calibration of the model variations with experimental data at mass ratio of 2.36 and validation are given in the next section.

\section{Results}

\subsection{Calibration and validation on the selected experimental arrangement}

In this study, 28 combinations of oscillators were calibrated using 8 schemes, which turned into more than 200 calibration runs, organized using parallel computing tools in Matlab. Experimental results obtained for mass ratio of 2.36 [2] have been chosen as an example of experimental data to perform the calibration procedure. As a result, 53 possible working sets of coefficients for different combinations of fluid nonlinearities were identified.

The results of this analysis are organized as three libraries containing the most accurate combinations of fluid nonlinearities with identified sets of coefficients and type of the statistical function that enables correct application of these options.

Library 1 is a set of the most accurate fluid nonlinearities and coefficient sets according to calibration procedure. These options were selected based on the lowest values of objective functions achieved during the calibration procedure, and also taking into account the sum of relative and absolute errors for all 26 points of the data for mass ratio of 2.36 in in-line and cross-flow directions without weighting. The model variations in this library are listed in Table 6 from the most accurate option at the top of the table to the least accurate option at the bottom of the table.

Table 6: Library 1: The most accurate model variations after calibration with low mass ratio of 2.36 [2].

\begin{tabular}{|c|l|l|l|}
\hline Option & Fluid nonlinearities & $\begin{array}{l}\text { Optimization } \\
\text { scheme }\end{array}$ & Set of coefficients \\
\hline 1 & Van der Pol - Rayleigh & Cross-flow 3 & $\begin{array}{l}C_{L 0}=1.87, C_{D 0}=2.31, C_{D 0}^{f l}=0.47, \varepsilon_{x}=2.1276, \varepsilon_{y}=0.0644, A_{x}= \\
12.24, A_{y}=3.78, C_{A}=4.06, K=0.94\end{array}$ \\
\hline 2 & Rayleigh - Rayleigh & Cross-flow 4 & $\begin{array}{l}C_{L 0}=0.85, C_{D 0}=2.41, C_{D 0}^{f l}=0.01, \varepsilon_{x}=0.4697, \varepsilon_{y}=0.0088, A_{x}= \\
11.98, A_{y}=6.90, C_{A}=3.12, K=0.78\end{array}$ \\
\hline 3 & Krenk-Nielsen & Cross-flow 4 & $\begin{array}{l}C_{L 0}=0.78, C_{D 0}=1.80, C_{D 0}^{f l}=0.64, \varepsilon_{x 1}=0.5946, \varepsilon_{x 2}= \\
0.0880, \varepsilon_{x 3}=0.9194, \varepsilon_{y}=0.0105, A_{x}=12.04, A_{y}=5.32, C_{A}= \\
1.69, K=0.83\end{array}$ \\
\hline 4 & Rayleigh Modified & In-line 4 & $\begin{array}{l}C_{L 0}=0.82, C_{D 0}=1.47, C_{D 0}^{f l}=0.01, \varepsilon_{x 1}=0.7055, \varepsilon_{x 2}= \\
1.0783, \varepsilon_{x 3}=0.7287, \varepsilon_{y 1}=0.1692, \varepsilon_{y 2}=0.0519, A_{x}=12.02, A_{y}=\end{array}$ \\
\hline
\end{tabular}




\begin{tabular}{|c|c|c|c|}
\hline 5 & Landl - Krenk-Nielsen & Cross-flow 4 & $\begin{array}{l}C_{L 0}=1.15, C_{D 0}=1.15, C_{D 0}^{f l}=0.03, \varepsilon_{x 1}=0.5186, \varepsilon_{x 2}= \\
0.5124, \varepsilon_{x 3}=0.5133, \varepsilon_{y 1}=0.0596, \varepsilon_{y 2}=0.0096, \varepsilon_{y 3}=0.2158, A_{x}= \\
11.96, A_{y}=4.11, C_{A}=1.30, K=0.42\end{array}$ \\
\hline 6 & Van der Pol - Rayleigh & In-line 4 & $\begin{array}{l}C_{L 0}=2.59, C_{D 0}=1.89, C_{D 0}^{f l}=0.11, \varepsilon_{x}=0.2244, \varepsilon_{y}=0.1770, A_{x}= \\
12.64, A_{y}=1.90, C_{A}=1.68, K=0.46\end{array}$ \\
\hline 7 & $\begin{array}{l}\text { Modified Van der Pol - } \\
\text { Modified Rayleigh }\end{array}$ & Cross-flow 3 & $\begin{array}{l}C_{L 0}=1.02, C_{D 0}=2.03, C_{D 0}^{f l}=0.22, \varepsilon_{x 1}=0.6651, \varepsilon_{x 2}= \\
0.6584, \varepsilon_{y 1}=0.0326, \varepsilon_{y 2}=0.0113, A_{x}=11.97, A_{y}=4.51, C_{A}= \\
2.42, K=1.19\end{array}$ \\
\hline 8 & $\begin{array}{l}\text { Landl - Modified } \\
\text { Rayleigh }\end{array}$ & Cross-flow 3 & $\begin{array}{l}C_{L 0}=0.98, C_{D 0}=1.95, C_{D 0}^{f l}=0.32, \varepsilon_{x 1}=1.0227, \varepsilon_{x 2}= \\
0.9989, \varepsilon_{x 3}=0.9521, \varepsilon_{y 1}=0.0591, \varepsilon_{y 2}=0.0097, A_{x}=11.98, A_{y}= \\
4.00, C_{A}=2.23, K=1.27\end{array}$ \\
\hline 9 & $\begin{array}{l}\text { Krenk-Nielsen - Krenk- } \\
\text { Nielsen }\end{array}$ & Cross-flow 3 & $\begin{array}{l}C_{L 0}=1.11, C_{D 0}=1.35, C_{D 0}^{f l}=0.82, \varepsilon_{x 1}=0.3952, \varepsilon_{x 2}= \\
0.4936, \varepsilon_{x 3}=0.7062, \varepsilon_{y 1}=0.0862, \varepsilon_{y 2}=0.0027, \varepsilon_{y 3}=0.0374, A_{x}= \\
11.99, A_{y}=2.96, C_{A}=1.03, K=1.22\end{array}$ \\
\hline
\end{tabular}

Comparative performance of the model variations from Library 1 presented in Table 6 is visualised in Figs $5-7$ for in-line and cross-flow amplitudes and corresponding frequencies along the range of reduced velocity of the flow $U_{R}$. Fig. 5 represents the calibration outcome for mass ratio of 2.36 in terms of displacements, while Fig. 6 illustrates frequencies of these responses. Fig. 7 shows the results of validation with the data collected on the same experimental facility for mass ratio of 3.68 [2] with no changes in the identified sets of coefficients.

In general, it is possible to observe, that all model variations from Library 1 when applied at mass ratio of 2.36 in Fig. 5 demonstrate significant underprediction of the lower branch of in-line lock-in state; and almost every option (except version 6) shows underprediction of the lower branch in cross-flow displacement amplitudes. It should be noted that our study also reveals that for mass ratio of 5.19, a significant discrepancies appear between the model predictions and the experimental data, and therefore it is concluded that the applicability of the identified model variations is limited to mass ratio from 2 to 5 . Above mass ratio of 5 , another set of coefficients or even different fluid nonlinearities might be beneficial to apply.

Option 1 from Library 1 given in Table 6 (Van der Pol - Rayleigh system calibrated using the scheme Crossflow 3 ) allows to predict both the largest amplitudes of resonance peaks in in-line and cross-flow directions at mass ratio of 2.36 (see Figs $5 \mathrm{a}$ and 5b). It demonstrates a slight overestimation of the initial branch of in-line lock-in and quite precise growth of super-upper branch until the top value. For the cross-flow direction, it shows some overestimation of the super-upper branch, but the drop from the top value to the lower branch is very accurate compared to the drops demonstrated by other options. This option is definitely advantageous in predicting both heights and widths of lock-in in both in-line and cross-flow directions.

Option 2 from Library 1 (Rayleigh - Rayleigh system calibrated with the scheme Cross-flow 4) allows to predict the largest in-line amplitude at mass ratio of 2.36 (see Figs 5a and 5b), but it appears slightly earlier in prediction than happens in reality. A similar situation is observed in the cross-flow direction, where the amplitude decrease starts at a lower velocity than it is observed in the experiment. However, it gives a more precise beginning of the lower branch than other oscillators predict, and less deviation from super-upper branch than Option 1 predicts. Also, this version of the model is simple, it has the smallest number of calibrated coefficients, as well as the original model with double classic Van der Pol equations and Option 1.

Version 3 from Library 1 (Krenk-Nielsen - Rayleigh system calibrated with the scheme Cross-flow 4) shows an overprediction of the largest in-line amplitude and at the lower reduced velocity than according to experimental data at mass ratio of 2.36 (see Figs $5 \mathrm{a}$ and $5 \mathrm{~b}$ ). In cross-flow direction, the peak is modelled very similar to Option 2 described above, this is why, their cross-flow curves are largely overlayed in Fig. 5b, with more visible 
(a)

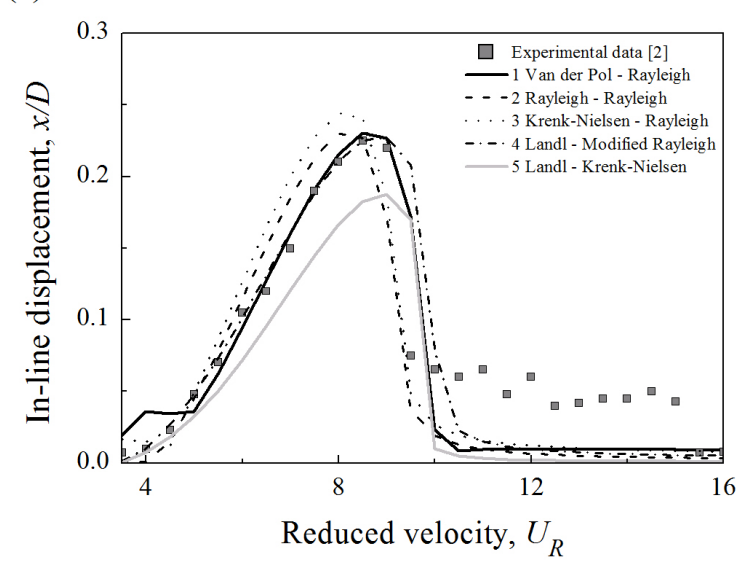

(c)

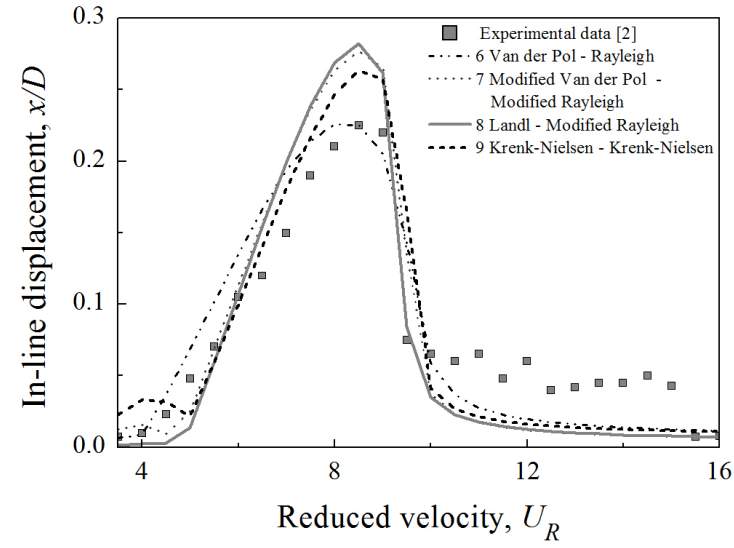

(b)

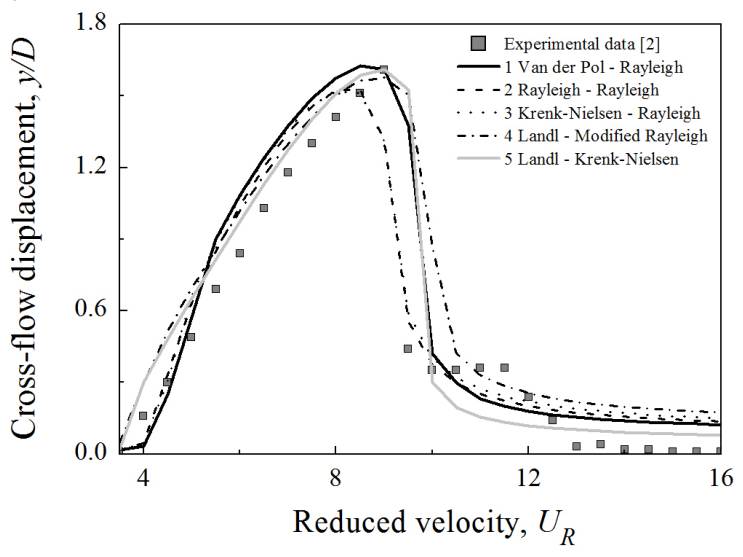

(d)

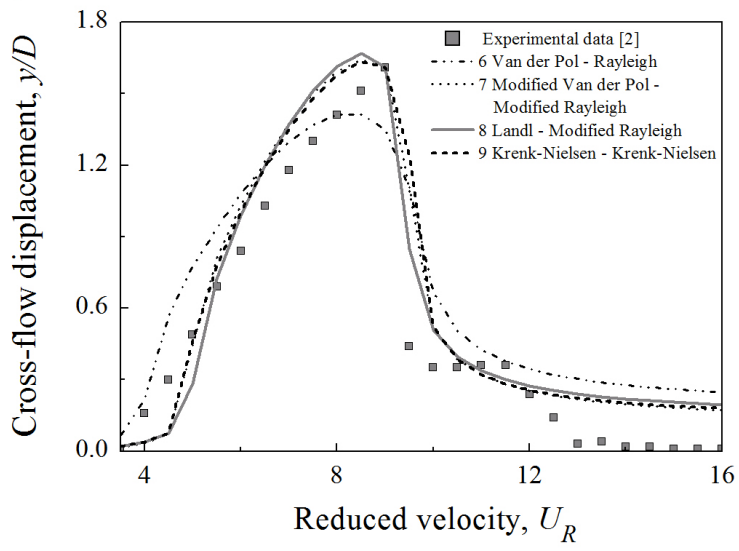

Figure 5: Displacement amplitudes during synchronisation state over the range of reduced velocity of the flow $U_{R}$ predicted by the models from Table 6 calibrated in respect to mass ratio of 2.36 in comparison with experimental data [2] for mass ratio 2.36: (a) prediction of in-line amplitudes of displacement for the model variations from 1 to 5; (b) prediction of cross-flow amplitudes of displacement for the model variations from 1 to 5; (c) prediction of in-line amplitudes of displacement for the model variations from 6 to 9; (d) prediction of cross-flow amplitudes of displacement for the model variations from 6 to 9 .

differences at the lower branch.

Version 4 from Library 1 (Landl - Modified Rayleigh system calibrated with the scheme In-line 4) is very precise describing growth of the super-upper branch in in-line direction at the mass ratio of 2.36 (see Figs $5 \mathrm{a}$ and $5 \mathrm{~b}$ ), but it predicts this branch to be slightly wider than in experiment. In cross-flow dimension, this option demonstrates a slight underestimation of the top value, overall wider resonance peak, and safer prediction in the region of lower branch.

Combination 5 from Library 1 (Landl - Krenk-Nielsen system calibrated with the scheme Cross-flow 4) shows a significant underprediction of the super-upper branch in in-line direction at the mass ratio of 2.36 (see Figs $5 \mathrm{a}$ and 5b). However, it has much smaller error with the super-upper branch in cross-flow direction, with correct largest observed amplitude, and a good safety margin in the range of reduced velocity from 3.5 to 10.0. Never the less, the lower branch gets more underestimated than using alternative options.

Combination 6 from Library 1 (Van der Pol - Rayleigh system calibrated with the scheme In-line 4) gives correct prediction of the top value in in-line dimension at mass ratio of 2.36 (see Figs $5 \mathrm{c}$ and $5 \mathrm{~d}$ ); an underestimation of the final point of the super-upper branch; and overall a wider resonance peak. Characteristics of the 
(a)

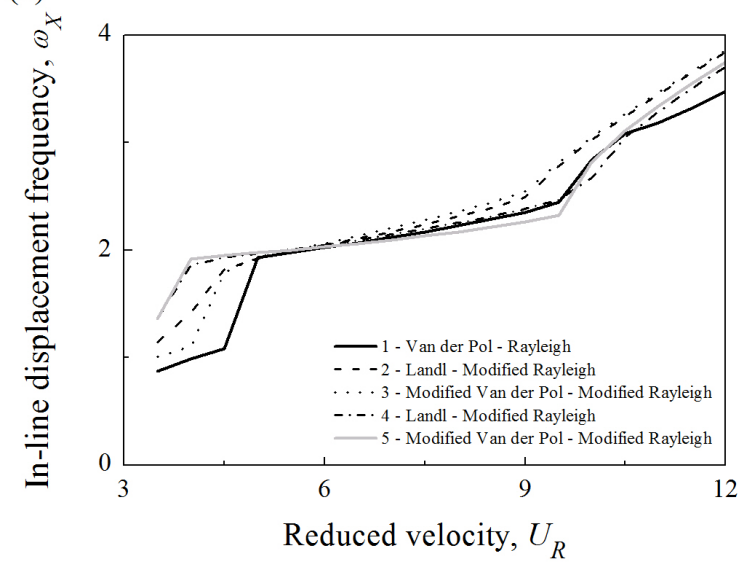

(c)

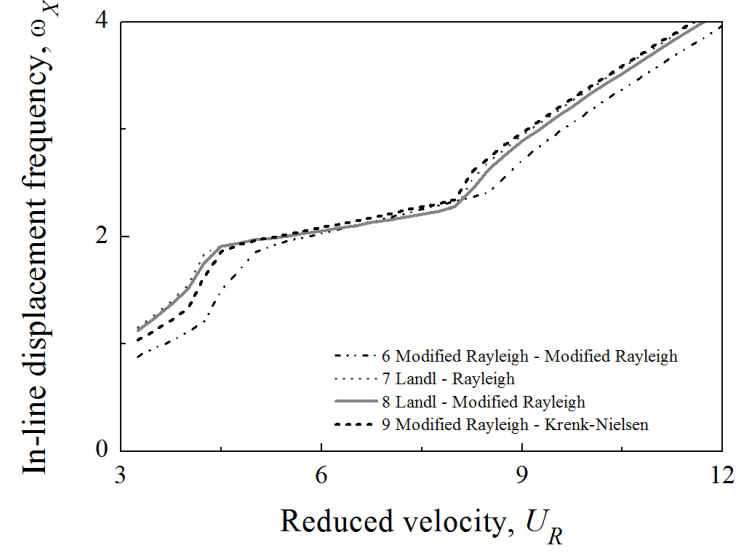

(b)

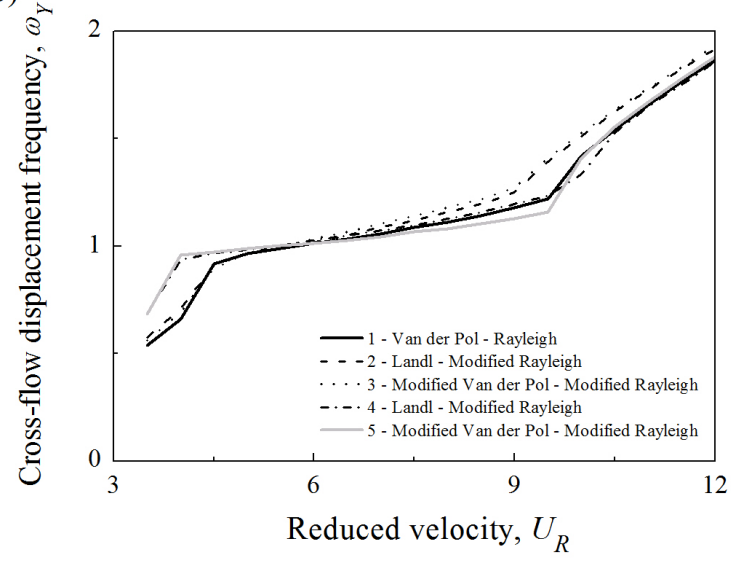

(d)

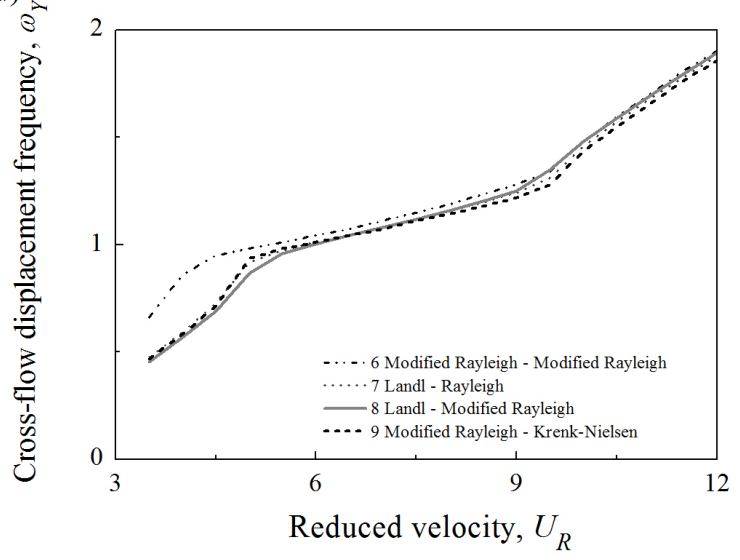

Figure 6: Displacement frequencies during synchronisation state over the range of reduced velocity of the flow $U_{R}$ predicted by the models from Table 6 calibrated in respect to mass ratio of 2.36: (a) frequencies of in-line displacement generated by the model variations from 1 to 5 ; (b) frequencies of cross-flow displacement generated by the model variations from 1 to 5 ; (c) frequencies of in-line displacement generated by the model variations from 6 to 9; (d) frequencies of cross-flow displacement generated by the model variations from 6 to 9 .

cross-flow peak are very much alike, but more pronounced: wider resonance peak than in real case; a significant underestimation of top cross-flow amplitudes. But this option is the only one that ensures a safe estimation of the lower branch in cross-flow direction, and is one of those which involves the smallest number of coefficients.

Model 7 from Library 1 (Modified Van der Pol - Modified Rayleigh system calibrated with the scheme Crossflow 3) shows a significant overestimation of the top in-line amplitude with a correct width of the resonance peak and a slight underestimation of initial branch at mass ratio of 2.36 (see Figs $5 \mathrm{c}$ and $5 \mathrm{~d}$ ). The super-upper branch in cross-flow lock-in is predicted with a safe margin, but the initial branch and lower branches are slightly underestimated.

Model 8 (Landl - Modified Rayleigh system calibrated with the scheme Cross-flow 3) and Model 9 (KrenkNielsen - Krenk-Nielsen system calibrated with the scheme Cross-flow 3) from Library 1 demonstrate the quality of fit at mass ratio of 2.36 (see Figs $5 \mathrm{c}$ and $5 \mathrm{~d}$ ) very similar to Model 7. However, Option 8 has the highest overestimation of the top of super-upper branch in both in-line and cross-flow directions, and very much realistic modelling of the drop from super-upper to lower branch in in-line direction.

In Fig. 6, it is shown that the applied oscillators lead to a different lock-in bandwidth in terms of the reduced velocity of the flow. Options 4 and 5 from Table 6 result in the longest lock-in around dimensionless 
(a)

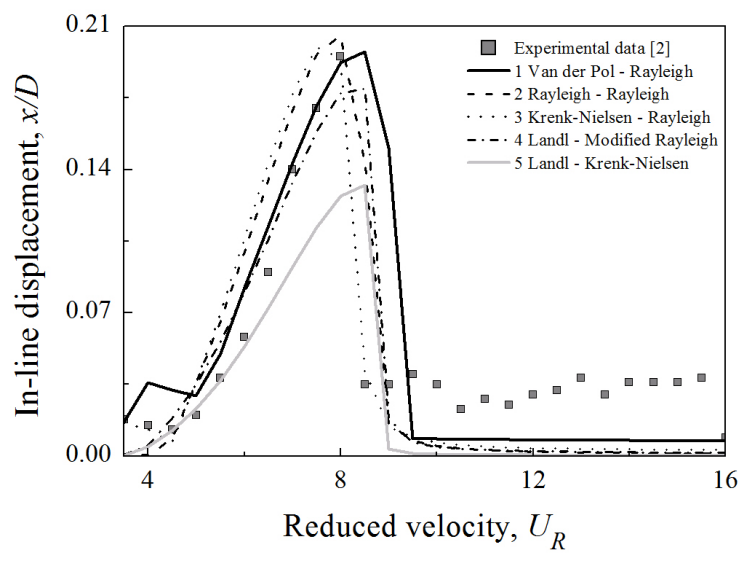

(c)

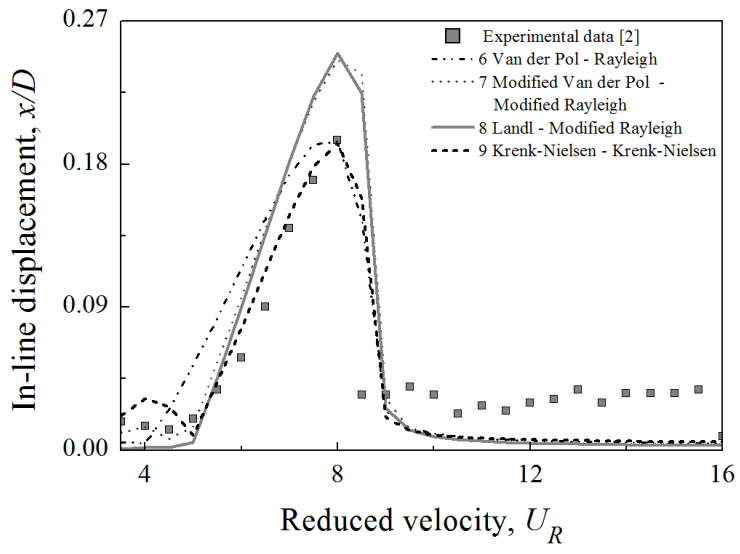

(b)

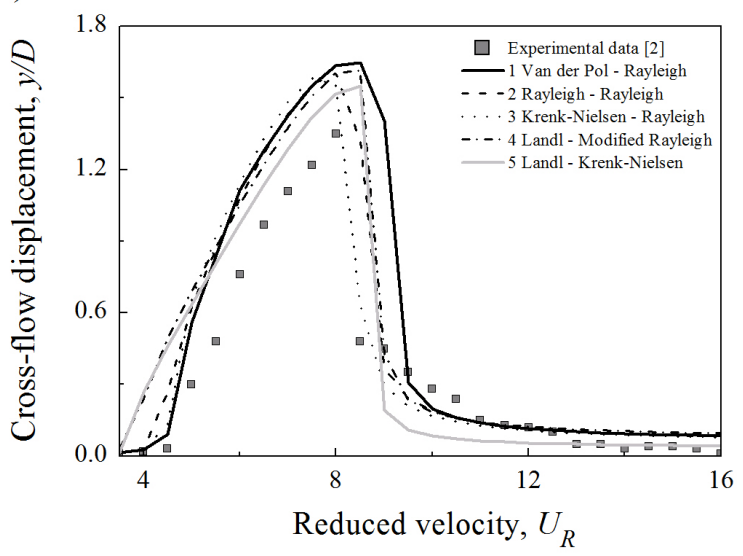

(d)

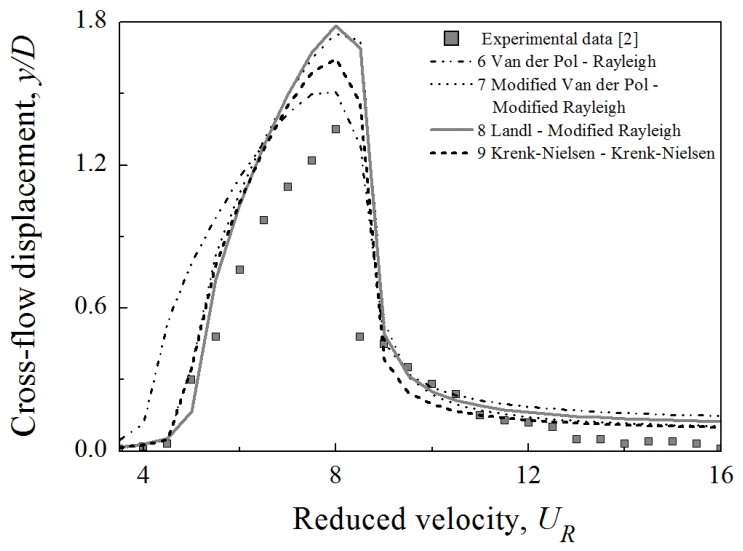

Figure 7: Displacement amplitudes during synchronisation state over the range of reduced velocity of the flow $U_{R}$ predicted by the models from Table 6 calibrated in respect to mass ratio of 2.36 and applied to mass ratio of 3.68 in comparison with experimental data [2] for mass ratio of 3.68: (a) prediction of the in-line amplitudes of displacement for the model variations from 1 to 5 ; (b) prediction of the cross-flow amplitudes of displacement for the model variations from 1 to 5 ; (c) prediction of the in-line amplitudes of displacement for the model variations from 6 to 9; (d) prediction of the cross-flow amplitudes of displacement for the model variations from 6 to 9 .

frequencies $\omega_{X}=2$ and $\omega_{Y}=1$ for in-line and cross-flow directions respectively. Here, dimensionless frequency in cross-flow direction is defined as $\omega_{Y}=\omega_{y} / \omega_{0}=\omega_{y} / \omega_{n}$, where $\omega_{y}$ is dimensional frequency of the cross-flow vibration, and the reference frequency $\omega_{0}$ is equal to the natural frequency of the structure $\omega_{n}$. In a similar manner, the in-line displacement frequency is $\omega_{X}=\omega_{x} / \omega_{0}=\omega_{x} / \omega_{n}$, with $\omega_{x}$ being dimensional frequency of the in-line displacement. Options 2 and 3 from Table 6 produce displacements of relatively higher frequencies in both directions before $U_{R}=5.0$ in comparison to the other model variations. Versions 6-8 from Table 6 generate in-line responses of relatively higher frequencies after the reduced velocity exceeds 7.0.

For mass ratio of 3.68, the obtained results are presented in Fig. 7. Option 9 from Library 1 given in Table 6 gives the most accurate prediction of the in-line displacement, including height and width of the peak. Models 2, 3, 7 and 8 deliver overpredictions of the top of in-line displacement peak; Models 1, 6 and 9 predict the largest amplitude exactly; and Options 4 and 5 underestimate the in-line displacement. For the cross-flow direction, all nine model variations overestimate the maximum peak value.

It is easy to notice that the working coefficients from Table 6 for the most accurate fit for set-up [2] were identified using the schemes Cross-flow 3, Cross-flow 4 and In-line 4. All of them include calculating the standard 
deviation of the displacement. Also, all fluid nonlinearities in Library 1 demonstrate that Rayleigh damping is present in every option as the necessary part of cross-flow equation. Even Krenk-Nielsen equation from Option 5 is a combination of Van der Pol and Rayleigh damping.

The most accurate models identified by the schemes with the maximum of displacement (Cross-flow 1 and 2) are listed in Table 7 as Library 1a. The accuracy of prediction using these options is disadvantageous when compared to Library 1.

The model variations and coefficients identified using the schemes In-line 1, 2 and 3, demonstrated worse predictions than those presented in Library 1 and Library 1a, and therefore they are not included in the paper.

Table 7: Library 1a: The most accurate models with appropriate coefficients after calibration with low mass ratio of 2.36 [2] using the optimization schemes with maximum of the generated displacements.

\begin{tabular}{|c|c|c|c|}
\hline Option & Fluid nonlinearities & $\begin{array}{l}\text { Optimization } \\
\text { scheme }\end{array}$ & Set of coefficients \\
\hline 1 & Van der Pol - Landl & Cross-flow 1 & $\begin{array}{l}C_{L 0}=1.03, C_{D 0}=1.51, C_{D 0}^{f l}=0.01, \varepsilon_{x}=0.4328, \varepsilon_{y 1}= \\
0.0297, \varepsilon_{y 2}=0.0211, \varepsilon_{y 3}=0.0099, A_{x}=12.21, A_{y}=4.10, C_{A}= \\
1.75, K=1.16\end{array}$ \\
\hline 2 & $\begin{array}{l}\text { Krenk-Nielsen - Krenk- } \\
\text { Nielsen }\end{array}$ & Cross-flow 1 & $\begin{array}{l}C_{L 0}=0.88, C_{D 0}=1.22, C_{D 0}^{f l}=0.0125, \varepsilon_{x 1}=0.5970, \varepsilon_{x 2}= \\
0.5874, \varepsilon_{x 3}=0.5738, \varepsilon_{y 1}=0.0195, \varepsilon_{y 2}=0.0199, \varepsilon_{y 3}=0.0964, A_{x}= \\
11.95, A_{y}=4.79, C_{A}=1.21, K=1.00\end{array}$ \\
\hline 3 & Rayleigh - Landl & Cross-flow 1 & $\begin{array}{l}C_{L 0}=1.13, C_{D 0}=1.34, C_{D 0}^{f l}=0.06, \varepsilon_{x}=0.1675, \varepsilon_{y 1}= \\
0.0727, \varepsilon_{y 2}=0.1964, \varepsilon_{y 3}=0.0607, A_{x}=10.92, A_{y}=3.67, C_{A}= \\
1.88, K=1.34\end{array}$ \\
\hline 4 & Rayleigh - Rayleigh & Cross-flow 2 & $\begin{array}{l}C_{L 0}=1.14, C_{D 0}=1.70, C_{D 0}^{f l}=0.03, \varepsilon_{x}=2.7484, \varepsilon_{y}=0.1441, A_{x}= \\
13.29, A_{y}=5.51, C_{A}=3.16, K=0.58\end{array}$ \\
\hline
\end{tabular}

\subsection{Validation with various experimental data}

To verify the chosen models with the selected coefficients, the published experimental data were used including the data by Jauvtis and Williamson [3] for mass ratio of 2.6; Srinil et al. [6] for mass ratio of 3.5; Blevins and Coughran [5] for mass ratio of 2.57 (according to the mass ratio definition in the current paper; in the original paper, mass ratio is $\left.\pi\left(\mu+C_{A}\right) / 4=2.8\right)$; and Dahl et al. [4] for in-line mass ratio of 3.3 and cross-flow mass ratio of 3.8. After preliminary validations using data obtained in these experimental set-ups, it was concluded that Library 1, which demonstrated a good, stable quality of the fit on the set-up by [2], is not fully suitable to predict VIVs at low mass ratio cases as the validation partly failed for different experimental arrangements. This is why, the authors of this study extended validation for other identified 44 fluid nonlinearities and working sets of coefficients. This led to a new list of model variations which are detailed in Table 8 as Library 2 .

It is worth reiterating here, that all modifications of the model in this paper were calibrated only once, and after that, were applied with no changes in the set of coefficients to explore the borders of application throughout different cases.

Library 2 contains fluid nonlinearities which are recommended by the authors of this paper as the most suitable for cases with mass ratio from 2 to 4 . This library was obtained based on visual selection of the accurate fit. It should be noted that this study does not aim to provide a rating of accuracy of the model variations, and that the model variations demonstrated some differences in performance when the modelling results were compared with experimental data obtained in different experimental set-ups. These are discussed in details below. 
Options 1 to 4 from Library 2 given in Table 8 appeared in the previous subsection as a part of Library 1 . Other options from 5 to 9 provide less accurate prediction for the set-up [2] but perform better for different experimental arrangements.

Table 8: Library 2: The model variations applicable at low mass ratio (from 2 to 4) on different experimental set-ups to predict both in-line and cross-flow responses.

\begin{tabular}{|c|c|c|c|c|}
\hline Option & Damping types & $\begin{array}{l}\text { Optimization } \\
\text { scheme }\end{array}$ & $\begin{array}{l}\text { Statistics } \\
\text { of signal }\end{array}$ & Set of coefficients \\
\hline 1 & $\begin{array}{l}\text { Van der Pol - } \\
\text { Rayleigh }\end{array}$ & Cross-flow 3 & $\begin{array}{l}\text { Standard } \\
\text { deviation }\end{array}$ & $\begin{array}{l}C_{L 0}=1.87, C_{D 0}=2.31, C_{D 0}^{f l}=0.47, \varepsilon_{x}=2.1276, \varepsilon_{y}= \\
0.0644, A_{x}=12.24, A_{y}=3.78, C_{A}=4.06, K=0.94\end{array}$ \\
\hline 2 & $\begin{array}{l}\text { Landl - Modified } \\
\text { Rayleigh }\end{array}$ & In-line 4 & $\begin{array}{l}\text { Standard } \\
\text { deviation }\end{array}$ & $\begin{array}{l}C_{L 0}=0.82, C_{D 0}=1.47, C_{D 0}^{f l}=0.01, \varepsilon_{x 1}=0.7055, \varepsilon_{x 2}= \\
1.0783, \varepsilon_{x 3}=0.7287, \varepsilon_{y 1}=0.1692, \varepsilon_{y 2}=0.0519, A_{x}= \\
12.02, A_{y}=5.92, C_{A}=1.24, K=0.41\end{array}$ \\
\hline 3 & $\begin{array}{l}\text { Modified } \text { Van der } \\
\text { Pol - Modified } \\
\text { Rayleigh }\end{array}$ & Cross-flow 3 & $\begin{array}{l}\text { Standard } \\
\text { deviation }\end{array}$ & $\begin{array}{l}C_{L 0}=1.02, C_{D 0}=2.03, C_{D 0}^{f l}=0.22, \varepsilon_{x 1}=0.6651, \varepsilon_{x 2}= \\
0.6584, \varepsilon_{y 1}=0.0326, \varepsilon_{y 2}=0.0113, A_{x}=11.97, A_{y}= \\
4.51, C_{A}=2.42, K=1.19\end{array}$ \\
\hline 4 & $\begin{array}{l}\text { Landl - Modified } \\
\text { Rayleigh }\end{array}$ & Cross-flow 3 & $\begin{array}{l}\text { Standard } \\
\text { deviation }\end{array}$ & $\begin{array}{l}C_{L 0}=0.98, C_{D 0}=1.95, C_{D 0}^{f l}=0.32, \varepsilon_{x 1}=1.0227, \varepsilon_{x 2}= \\
0.9989, \varepsilon_{x 3}=0.9521, \varepsilon_{y 1}=0.0591, \varepsilon_{y 2}=0.0097, A_{x}= \\
11.98, A_{y}=4.00, C_{A}=2.23, K=1.27\end{array}$ \\
\hline 5 & $\begin{array}{l}\text { Modified Van der } \\
\text { Pol - Modified } \\
\text { Rayleigh }\end{array}$ & Cross-flow 4 & $\begin{array}{l}\text { Standard } \\
\text { deviation }\end{array}$ & $\begin{array}{l}C_{L 0}=0.81, C_{D 0}=1.71, C_{D 0}^{f l}=0.01, \varepsilon_{x 1}=0.5979, \varepsilon_{x 2}= \\
0.5964, \varepsilon_{y 1}=0.0137, \varepsilon_{x 2}=0.0109, A_{x}=11.96, A_{y}= \\
4.58, C_{A}=1.19, K=0.59\end{array}$ \\
\hline 6 & $\begin{array}{ll}\text { Modified } & \text { Rayleigh } \\
- & \text { Modified } \\
\text { Rayleigh } & \\
\end{array}$ & Cross-flow 3 & $\begin{array}{l}\text { Standard } \\
\text { deviation }\end{array}$ & $\begin{array}{l}C_{L 0}=0.77, C_{D 0}=2.12, C_{D 0}^{f l}=0.20, \varepsilon_{x 1}=0.7046, \varepsilon_{x 2}= \\
0.7270, \varepsilon_{y 1}=0.0125, \varepsilon_{y 2}=0.0042, A_{x}=11.95, A_{y}= \\
5.27, C_{A}=1.84, K=1.04\end{array}$ \\
\hline 7 & Landl - Rayleigh & Cross-flow 4 & $\begin{array}{l}\text { Standard } \\
\text { deviation }\end{array}$ & $\begin{array}{l}C_{L 0}=0.90, C_{D 0}=1.41, C_{D 0}^{f l}=0.01, \varepsilon_{x 1}=0.6497, \varepsilon_{x 2}= \\
0.6350, \varepsilon_{x 3}=0.6414, \varepsilon_{y}=0.0234, A_{x}=11.95, A_{y}= \\
3.88, C_{A}=0.93, K=0.48\end{array}$ \\
\hline 8 & $\begin{array}{l}\text { Landl - Modified } \\
\text { Rayleigh }\end{array}$ & Cross-flow 2 & Maximum & $\begin{array}{l}C_{L 0}=0.79, C_{D 0}=1.42, C_{D 0}^{f l}=0.24, \varepsilon_{x 1}=1.5007, \varepsilon_{x 2}= \\
0.8436, \varepsilon_{x 3}=1.2897, \varepsilon_{y 1}=0.0160, \varepsilon_{y 2}=0.0218, A_{x}= \\
11.60, A_{y}=4.49, C_{A}=0.76, K=0.55\end{array}$ \\
\hline 9 & $\begin{array}{l}\text { Modified Rayleigh } \\
\text { - Krenk-Nielsen }\end{array}$ & Cross-flow 3 & $\begin{array}{l}\text { Standard } \\
\text { deviation }\end{array}$ & $\begin{array}{l}C_{L 0}=0.57, C_{D 0}=1.33, C_{D 0}^{f l}=0.15, \varepsilon_{x 1}=0.4020, \varepsilon_{x 2}= \\
0.3812, \varepsilon_{y 1}=0.0288, \varepsilon_{y 2}=0.00005, \varepsilon_{y 3}=0.0062, A_{x}= \\
11.97, A_{y}=4.83, C_{A}=0.35, K=0.57\end{array}$ \\
\hline
\end{tabular}

The next figures show results of the fit with the data from different experimental arrangements. A comparison with the data by Jauvtis and Williamson [3] for mass ratio of 2.6 is presented in Fig. 8. For in-line lock-in in Figs $8 \mathrm{a}$ and $8 \mathrm{c}$, the most accurate prediction of the peak height is provided by Option 8 from Library 2 (Landl - Modified Rayleigh system calibrated with the scheme Cross-flow 2). The safe fit is also ensured by Option 9 (Modified Rayleigh - Krenk-Nielsen system calibrated with the scheme Cross-flow 3). All options from Library 2 do not predict development of the first in-line lock-in at reduced velocity of 2.5 when applied to the case in [3] with mass ratio of 2.6. It is clear that this is caused by the data [2] which were utilised to calibrate the model as they contained only the range from reduced velocity of 3.5. This means that, ideally, calibration with in-line direction should be accomplished using the data with more extended range of reduced velocity, starting from approximately 1.5. Also, all the considered options are not capable to model the lower branch of in-line peak correctly, which was the problem during the validations with the data [2] as well.

For cross-flow lock-in shown in Figs 8b and 8d, a safe fit is provided by Option 1 (Van der Pol - Rayleigh 
(a)

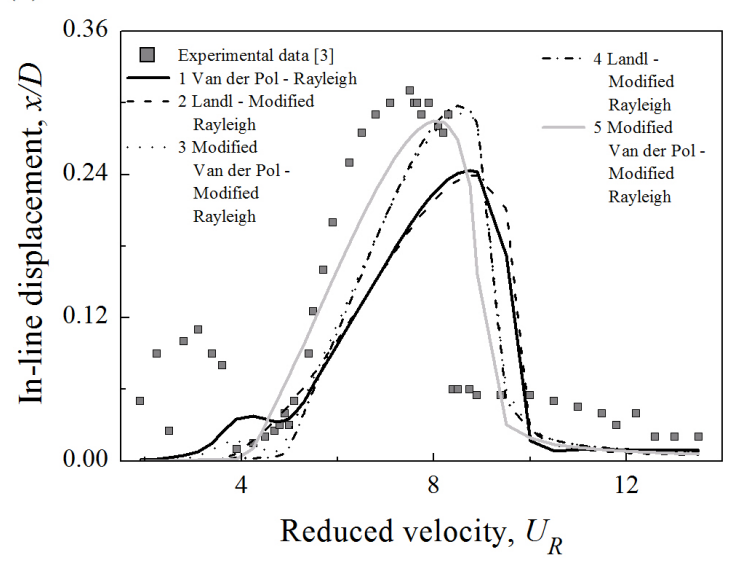

(c)

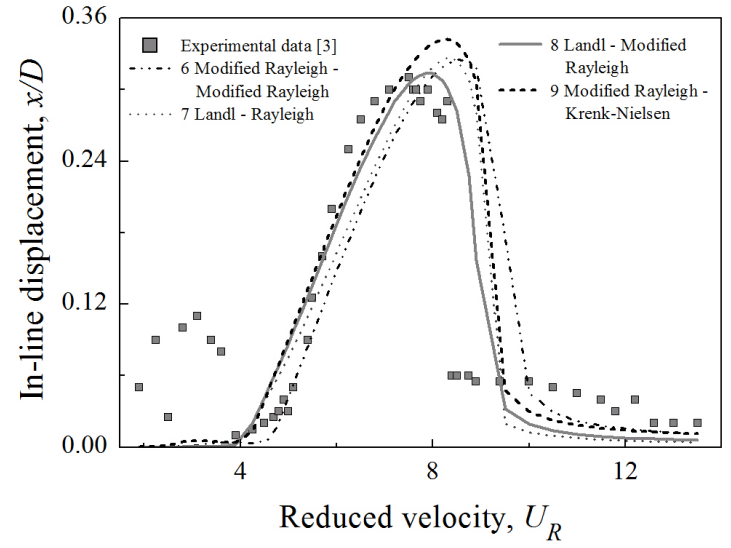

(e)

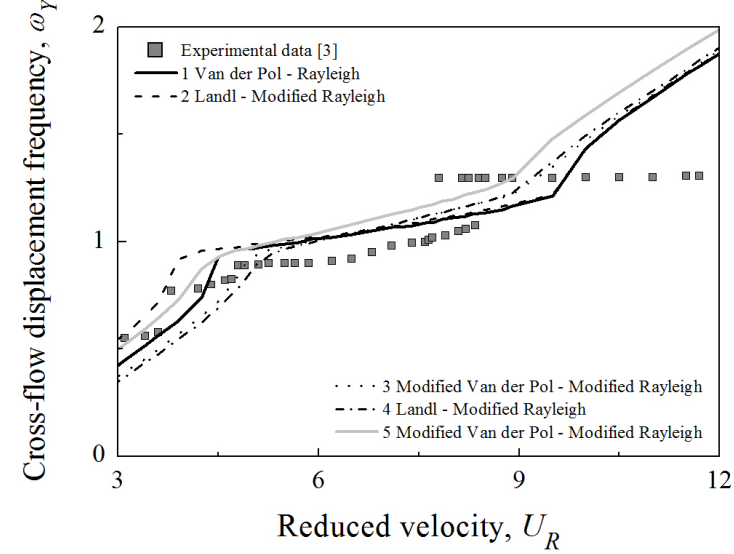

(b)

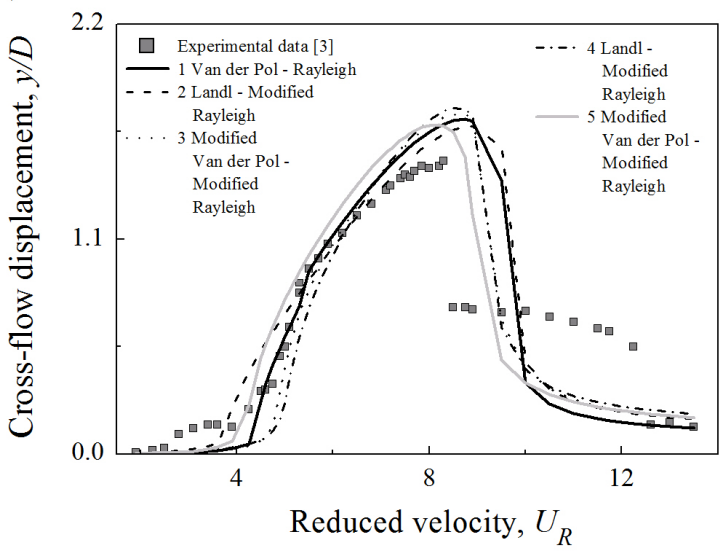

(d)

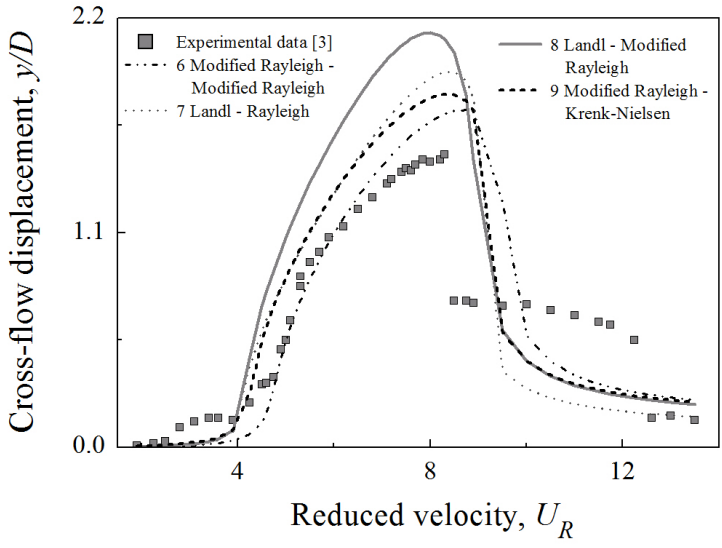

(f)

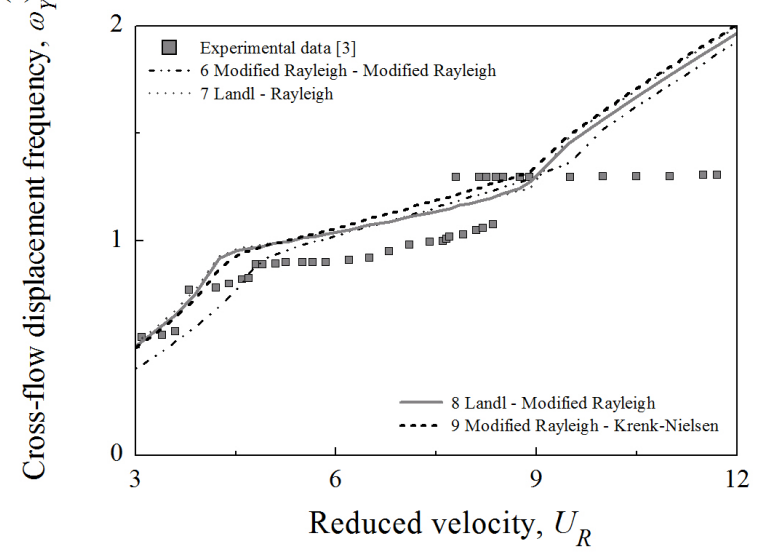

Figure 8: Experimental data by Jauvtis and Williamson [3] for mass ratio of 2.6 against displacement amplitudes predicted by the model variations of Library 2 from Table 8: (a) in-line displacement amplitudes predicted by Options from 1 to 5; (b) cross-flow displacement amplitudes predicted by Options from 1 to 5; (c) in-line displacement amplitudes predicted by Options from 6 to 9; (d) cross-flow displacement amplitudes predicted by Options from 6 to 9; (e) cross-flow displacement frequencies predicted by Options from 1 to 5 ; (f) cross-flow displacement frequencies predicted by Options from 6 to 9 .

system calibrated with the scheme Cross-flow 3), Option 5 (Modified Van der Pol - Modified Rayleigh system calibrated with the scheme Cross-flow 4), Option 7 (Landl - Rayleigh system calibrated with the scheme Crossflow 4) and Option 9 (Modified Rayleigh - Krenk-Nielsen system calibrated with the scheme Cross-flow 3). 
(a)

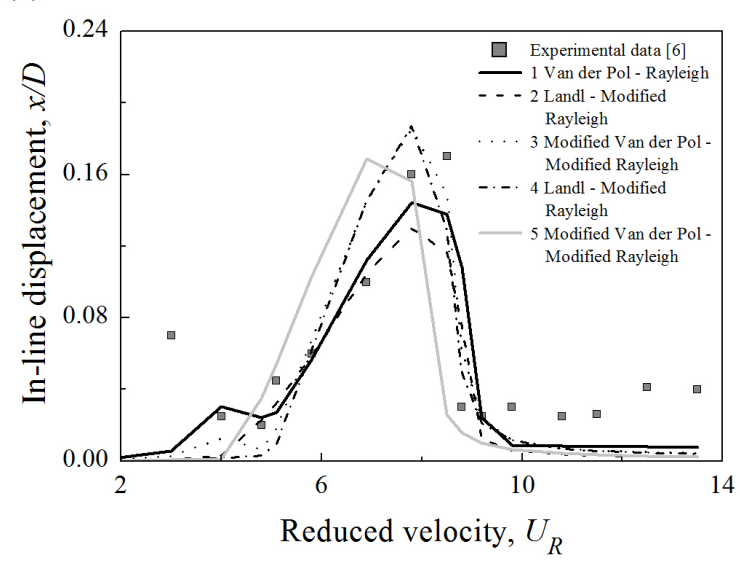

(c)

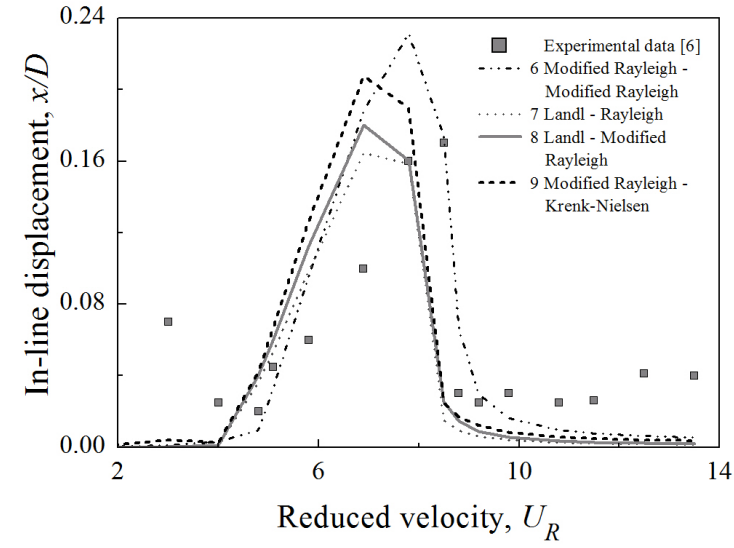

(b)

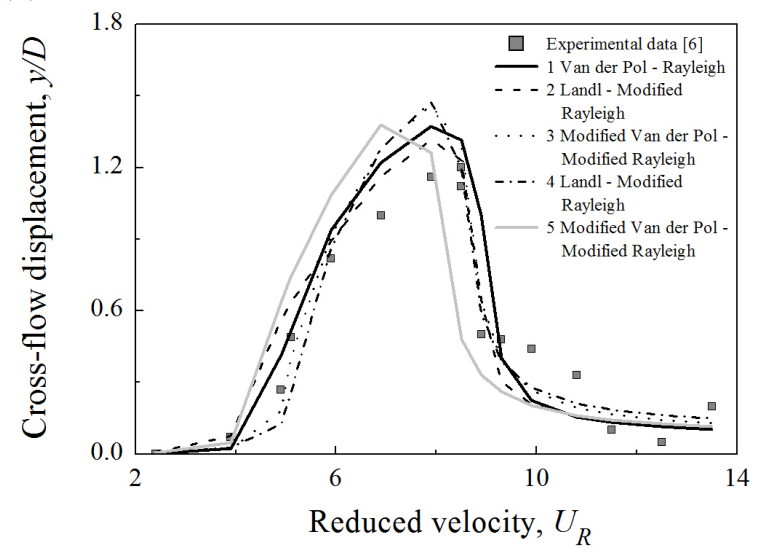

(d)

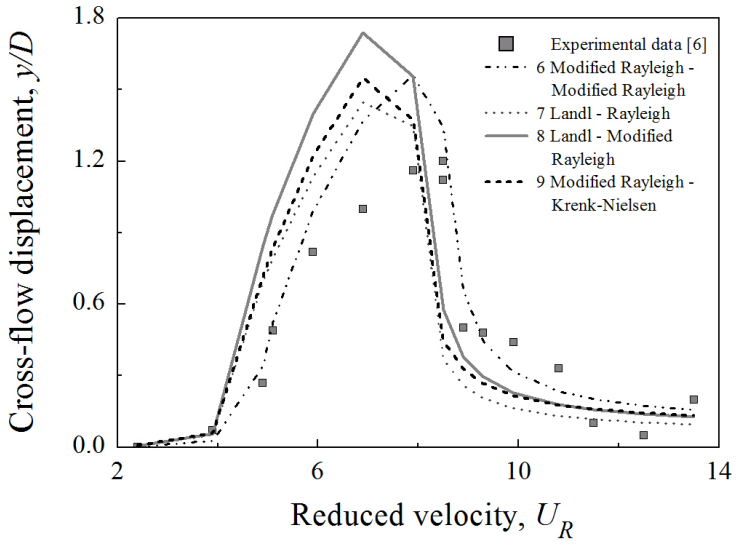

Figure 9: Experimental data by Srinil et al. [6] (test KHL6 in the original paper) obtained as root mean square of displacement amplitudes for mass ratio of 3.5 against displacement amplitudes predicted by the model variations of Library 2 from Table 8: (a) in-line displacement amplitudes predicted by Options from 1 to 5 ; (b) cross-flow displacement amplitudes predicted by Options from 1 to 5; (c) in-line displacement amplitudes predicted by Options from 6 to 9; (d) cross-flow displacement amplitudes predicted by Options from 6 to 9 .

Variant 8 from Library 2 gives a significant overestimation of the resonance development, and other model variations underpredict the super-upper branch. The main general disadvantage of the fit of cross-flow response here is underestimation of the lower branch.

In Figs 8e and 8f, the frequencies of generated displacements for cross-flow direction are predicted to be generally higher than appears in experimental data [3]. Among the considered fluid nonlinearities, Option 1 seems to be qualitatively closer to the experimentally observed frequencies of super-upper branch. Options 1 , $2,5,7,8,9$ give relatively accurate frequencies of the displacement signals at $U_{R}=3.0-4.0$. All the considered oscillators overestimate the frequencies of the displacements corresponding to the lower branch after $U_{R}=9.0$.

A validation with the data by Srinil et al. [6] for mass ratio of 3.5 given in Fig. 9 leads to the conclusion that the best fit here is delivered by Models 1, 2, 3, 4, and 6 from Library 2. This result is very much alike as the options suitable for the arrangement by Stappenbelt and Lalji [2].

A successful prediction of the resonance state in the cases described in Dahl et al. [4] and Blevins and Coughran [5] was obtained in this study only for the cross-flow direction, as shown in Figs 10 and 11 respectively.

The cross-flow lock-in estimation in Figs 10a and 10b for in-line mass ratio of 3.3 and cross-flow mass ratio of 3.8 is the most accurate using the fluid nonlinearities $5,7,8$ and 9 from Library 2 due to the correct 
(a)

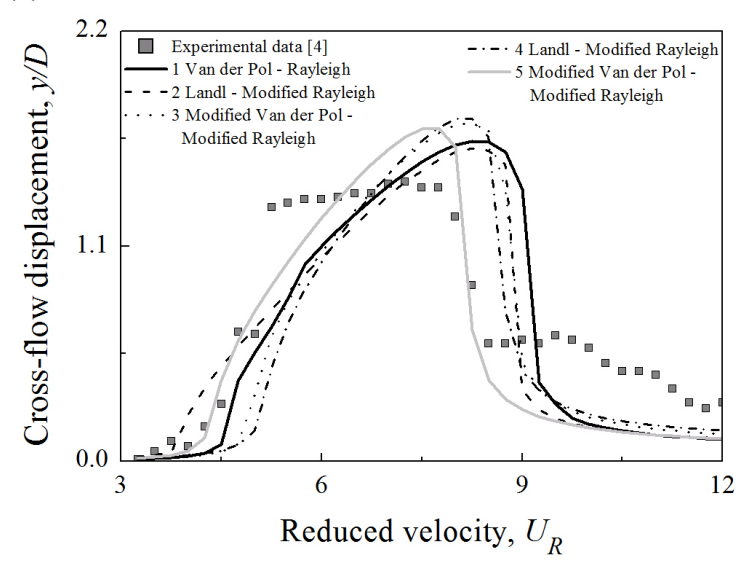

(c)

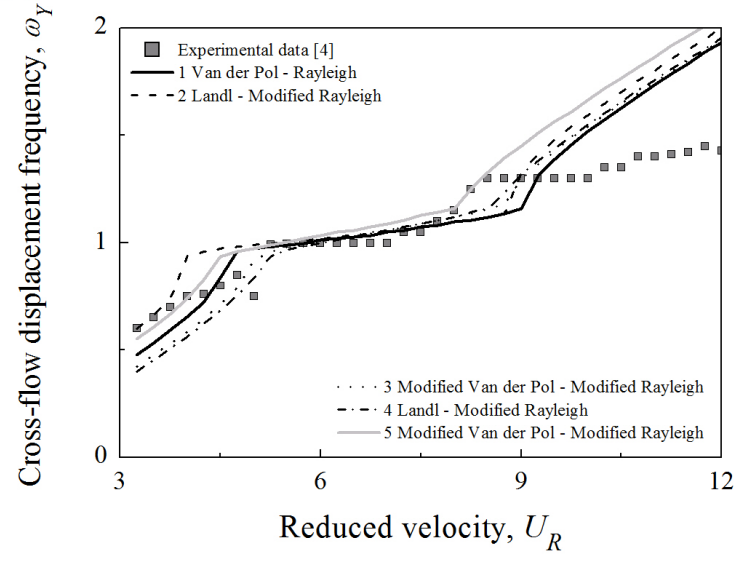

(b)

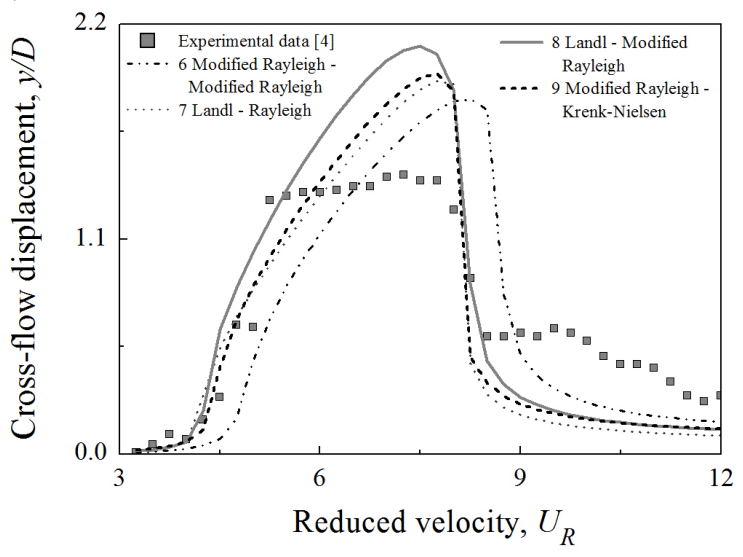

(d)

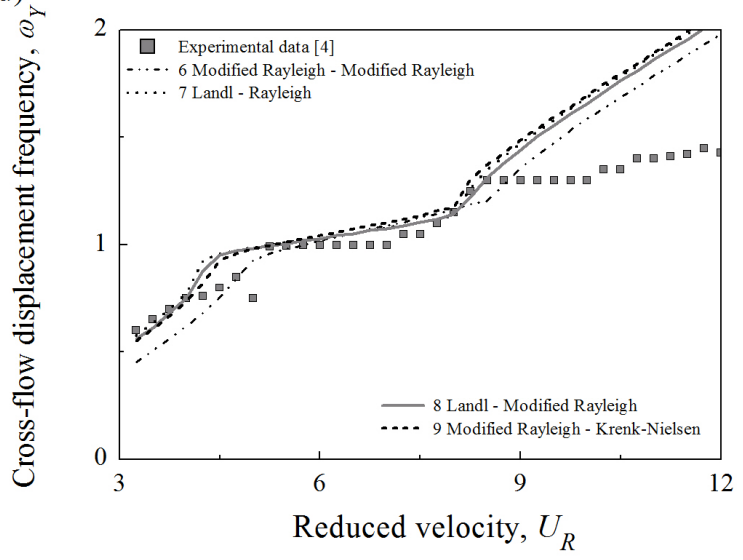

Figure 10: Experimental data by Dahl et al. [4] (test 1 in the original paper with frequency ratio $f^{*}=1.00$ ) for in-line mass ratio of 3.3 and cross-flow mass ratio of 3.8 against displacement amplitudes predicted by the model variations of Library 2 from Table 8: (a) cross-flow displacement amplitudes predicted by Options from 1 to 5; (b) cross-flow displacement amplitudes predicted by Options from 6 to 9; (c) cross-flow displacement frequencies predicted by Options from 1 to 5; (d) cross-flow displacement frequencies predicted by Options from 6 to 9 .

determination of the width of the super-upper branch. Option 8 can be considered as the safest variant due to almost correct reconstruction of transition from the initial to super-upper branch in the reduced velocity interval from 4 to 5 . The estimation of dimensionless cross-flow displacement frequency shown in Figs 10c and $10 \mathrm{~d}$ varies in accuracy depending on $U_{R}$. Thus, the best fit for the central lock-in region from 5.0 to 7.5 is provided by the model variations $1-4$ from Table 8 . The model versions 5 and 8 provide adequate frequency response of the cylinder around $U_{R}=8.0$. All the considered combinations of fluid nonlinearities generate signal frequencies higher than experimental after $U_{R}=9.0$. Options $2,5,7,8,9$ provide a respectable fit of the frequency response at the region of reduced velocity $U_{R}=3.0-4.0$. The region of the reduced velocity from 4.0 to 5.0 possesses the experimental frequencies which are not reproduced by the considered oscillators from Table 8 .

The cross-flow synchronisation fit in Fig. 11 for mass ratio of 2.57 is the most successful when applying Modification 8 from Library 2 as well. This model version allows very close prediction of the top of super-upper branch. Options 9 and 7 can be seen as the second most accurate model variations. Option 6 from Library 2 deserves attention because it enables the safest prediction of the lower branch and almost exact modelling of transition from the initial to the super-upper branch. 
(a)

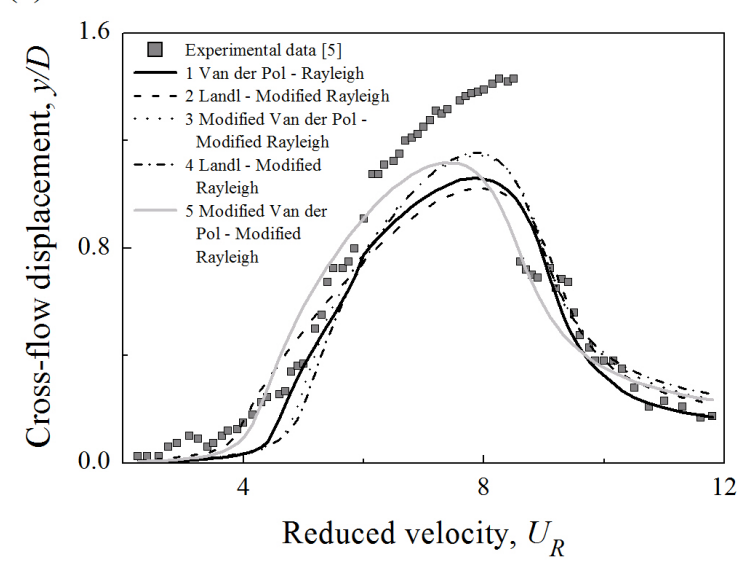

(b)

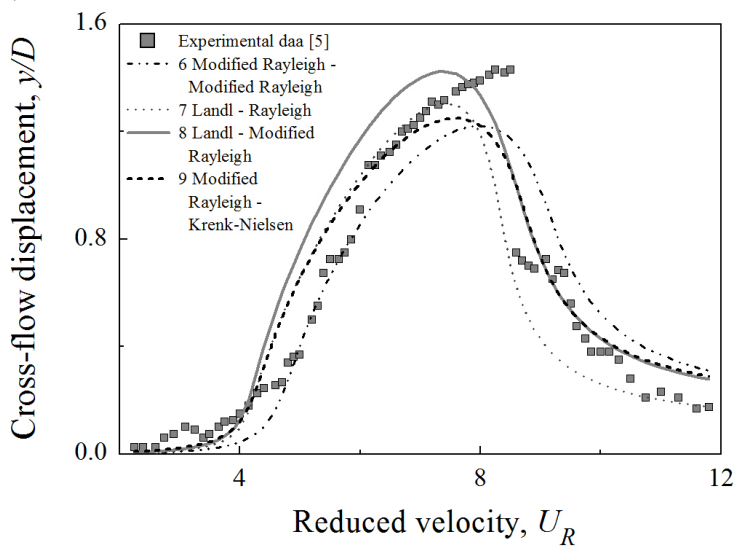

Figure 11: Experimental data by Blevins and Coughran [5] for mass ratio of 2.57 against displacement amplitudes predicted by the model variations of Library 2 from Table 8: (a) cross-flow displacement amplitudes predicted by Options from 1 to 5; (b) cross-flow displacement amplitudes predicted by Options from 6 to 9 .

A comparison with the various sources of experimental data shows that the model variations from 1 to 4 from Library 2 show a better quality of prediction on the set-up by Stappenbelt and Lalji [2] they were calibrated with, and also for the data by Srinil et al. [6]. The model variations from 5 to 9 from Library 2 give significant overestimations for the set-up [2], but they are more reliable for other experimental set-ups: Dahl et al. [4] and Blevins and Coughran [5]. For the data by Jauvtis and Williamson [3], Options 1, 5, 7 and 9 are better performing for cross-flow peak prediction, and Options 8 and 9 are better for in-line direction.

Overall, the advised application range for Library 2 is mass ratio interval from 2 to 4 to provide a better accuracy of prediction in height and width of the resonance peak of displacement amplitudes. All nonlinearities from Library 2 can be applied to equip other wake oscillator models to work with low mass ratio cases.

Analysing the second and third columns from Tables 6 and 8 together allows to make the following observations:

1. Majority of the most accurate options are provided by standard deviation of the generated signals of displacements that corresponds to the calibration schemes Cross-flow 3, Cross-flow 4, In-line 4.

2. One scheme of optimization is not enough to identify all the best sets of coefficients. It is essential to conduct calibration using at least 2 different logical algorithms, otherwise, some good sets of coefficients can be missing.

3. Van der Pol damping appears very often to describe in-line motion of the low mass ratio structure. Van der Pol damping is a part of Landl equation and Krenk-Nielsen equation which can be suitable for this purpose.

4. Rayleigh damping features in all options as a form of the cross-flow equation. Rayleigh damping is also a part of Krenk-Nielsen equation.

\section{Conclusions and future work}

In this work, a suite of VIV wake oscillator models was investigated where the models differ in types of nonlinear damping included in the fluid equations, and the impact of nonlinear damping types on the accuracy of VIV 
prediction was considered. The model variations were calibrated using the data by Stappenbelt and Lalji [2] for "low" mass ratio of 2.36, and then, validated with other sources of experimental data [3-6]. The sets of model coefficients were optimized for each variation of the nonlinear damping using the constrained nonlinear minimization. The results of this study are presented as two main libraries of the model variations with the best identified sets of coefficients. The obtained libraries are briefly characterized in Table 9. As can be seen, the options from Library 2 can model the behaviour of 2DOFs rigid structure in different experimental arrangements if the mass ratio of the structure is between 2 and 4, and they can be considered as the most successful outcome of this study.

Table 9: Summary of the model libraries obtained in this research for low mass ratio structures

\begin{tabular}{|c|c|c|c|}
\hline $\begin{array}{c}\text { Number of } \\
\text { the library }\end{array}$ & $\begin{array}{c}\text { Number of } \\
\text { model variations }\end{array}$ & $\begin{array}{c}\text { Number of } \\
\text { identified sets of } \\
\text { coefficients }\end{array}$ & Applicability \\
\hline 1 & 7 & 9 & $\begin{array}{c}\text { Valid for mass ratio from 2 to } 5 \text { on the set-up by } \\
\text { Stappenbelt and Lalji }[2]\end{array}$ \\
\hline 2 & 6 & 9 & $\begin{array}{c}\text { Valid for mass ratio from 2 to } 4 \text { on five different } \\
\text { experimental set-ups }[2-6]\end{array}$ \\
\hline
\end{tabular}

The frequency difference between the beginning of resonance computed by the base model [1] and the experimental data [2] (the lock-in delay coefficient $K$ ) was identified as an additional coefficient for calibration, and for the majority of cases it was found to be in the range $K \in(0.5,1.5)$ for the low mass ratio from 2 to 5 . Results shown in Figs 8 - 11 allow to confirm that lock-in delay coefficient $K$ identified once for the set-up by Stappenbelt and Lalji [2] at mass ratio of 2.36 is valid for other experimental facilities for two degrees-of-freedom rigid structures. However, this parameter, along with the other found empirical coefficients, can be sensitive to the experimental set-up arrangements which are not reflected in the base model. This issue will be a subject of further study.

Analysis of frequencies of the generated displacements demonstrates that the considered oscillators allow relatively accurate predictions of frequencies observed in experiments by [3,4] for the initial and super-upper branches of the resonance in cross-flow direction. However, the displacement frequencies after $U_{R}$ of 9.0 are significantly overestimated.

It is important to stress out here, that performing calibration of wake oscillator models using different strategies can significantly improve the model predictions. When the number of calibrated parameters is high (9 and more in the current study), sets of coefficients should be calibrated with distinguished algorithms, to avoid missing the viable options. The conducted analysis reveals that the Rayleigh damping is the most suitable for cross-flow equation, whereas the Van der Pol damping is preferable for in-line equation for the low mass ratio cases.

Another way to continue improving wake oscillator models is to consider designing synthetic oscillators for particular cases. But this task would require, at first, detailed studies of the limitations of already existing models using experimental data for comparable cases.

The analysis and calibration of the models considered in the current work were focused on the cases with low mass ratios. Our preliminary study indicates that it would be beneficial to conduct similar investigations for medium and high mass ratios so the best options with accurately identified sets of coefficients are available for the whole range of the cylinder properties and they could be used in the design of the offshore structures subjected to VIVs. 


\section{Acknowledgements}

V.K. would like to acknowledge the support of the Industrial University of Tyumen, Tyumen, Russia, and the State Program "Global Education", Russia. This work has been performed using the Maxwell High Performance Computing Cluster funded by the University of Aberdeen. Authors would like to express their gratitude to Dr Andrew Starkey for advices regarding optimization procedures and to Naveed Khan for technical advices on accelerating computations.

\section{References}

[1] A. Postnikov, E. Pavlovskaia, and M. Wiercigroch. 2DOF CFD calibrated wake oscillator model to investigate vortex-induced vibrations. International Journal of Mechanical Sciences, 127:176-190, 2017.

[2] B. Stappenbelt and F. Lalji. Vortex-induced vibration super-upper response branch boundaries. International Journal of Offshore and Polar Engineering, 18(02):99-105, 2008.

[3] N. Jauvtis and C.H.K. Williamson. The effect of two degrees-of-freedom on vortex-induced vibration at low mass and damping. Journal of Fluid Mechanics, 509:23-62, 2004.

[4] J.M. Dahl, F.S. Hover, and M.S. Triantafyllou. Two-degree-of-freedom vortex-induced vibrations using a force assisted apparatus. Journal of Fluids and Structures, 22(6):807-818, 2006.

[5] R.D. Blevins and C.S. Coughran. Experimental investigation of vortex-induced vibration in one and two dimensions with variable mass, damping, and Reynolds number. Journal of Fluids Engineering, 131(10): $101202,2009$.

[6] N. Srinil, H. Zanganeh, and A. Day. Two-degree-of-freedom VIV of circular cylinder with variable natural frequency ratio: Experimental and numerical investigations. Ocean Engineering, 73:179-194, 2013.

[7] W.D. Iwan and R.D. Blevins. A model for vortex-induced oscillation of structures. Journal of Applied Mechanics, 41(3):581-586, 1974.

[8] H. Zanganeh and N. Srinil. Three-dimensional VIV prediction model for a long flexible cylinder with axial dynamics and mean drag magnifications. Journal of Fluids and Structures, 66:127-146, 2016.

[9] X. Bai and W. Qin. Using vortex strength wake oscillator in modelling of vortex induced vibrations in two degrees-of-freedom. European Journal of Mechanics-B/Fluids, 48:165-173, 2014.

[10] A. Roshko. On the wake and drag of bluff bodies. Journal of the Aeronautical Sciences, 22(2):124-132, 1955.

[11] R.E.D. Bishop and A.Y. Hassan. The lift and drag forces on a circular cylinder oscillating in a flowing fluid. Proceedings of the Royal Society of London A: Mathematical, Physical and Engineering Sciences, 277 (1368):51-75, 1964.

[12] R.T. Hartlen and I.G. Currie. Lift-oscillator model of vortex-induced vibration. Journal of the Engineering Mechanics Division, 96(5):577-591, 1970.

[13] R. Landl. A mathematical model for vortex-excited vibrations of bluff bodies. Journal of Sound and Vibration, 42(2):219-234, 1975. 
[14] S. Krenk and S.R.K. Nielsen. Energy balanced double oscillator model for vortex-induced vibrations. Journal of Engineering Mechanics, 125(3):263-271, 1999.

[15] W.-J. Kim and N.C. Perkins. Two-dimensional vortex-induced vibration of cable suspensions. Journal of Fluids and Structures, 16(2):229-245, 2002.

[16] A.H. Nayfeh, F. Owis, and M.R. Hajj. A model for the coupled lift and drag on a circular cylinder. ASME 2003 International Design Engineering Technical Conferences and Computers and Information in Engineering Conference. pp. 1289-1296, 2003.

[17] J.M.O. Barbosa, Y. Qu, A. Metrikine, and E.-M. Lourens. Vortex-induced vibrations of a freely vibrating cylinder near a plane boundary: Experimental investigation and theoretical modelling. Journal of Fluids and Structures, 69:382-401, 2017.

[18] M.L. Facchinetti, E. De Langre, and F. Biolley. Coupling of structure and wake oscillators in vortex-induced vibrations. Journal of Fluids and structures, 19(2):123-140, 2004.

[19] R.H.M. Ogink and A.V. Metrikine. A wake oscillator with frequency dependent tuning coefficients for the modeling of VIV. ASME 2008 27th International Conference on Offshore Mechanics and Arctic Engineering. pp. 943-952, 2008.

[20] F. Ge, X. Long, L. Wang, and Y. Hong. Flow-induced vibrations of long circular cylinders modeled by coupled nonlinear oscillators. Science in China Series G: Physics, Mechanics and Astronomy, 52(7):10861093, 2009.

[21] R.H.M. Ogink and A.V. Metrikine. A wake oscillator with frequency dependent coupling for the modeling of vortex-induced vibration. Journal of Sound and Vibration, 329(26):5452-5473, 2010.

[22] E. Pavlovskaia, M. Keber, A. Postnikov, K. Reddington, and M. Wiercigroch. Multi-modes approach to modelling of vortex-induced vibration. International Journal of Non-Linear Mechanics, 80:40-51, 2016.

[23] G. Moe and Z.J. Wu. The lift force on a cylinder vibrating in a current. Transactions of the ASME. Journal of Offshore Mechanics and Arctic, 112(4):297-303, 1990.

[24] G. Moe, K. Holden, and P.O. Yttervoll. Motion of spring supported cylinders in subcritical and critical water flows. The Fourth International Offshore and Polar Engineering Conference. pp. 468-475, 1994.

[25] M.R. Gharib. Vortex-induced vibration, absence of lock-in and fluid force deduction. PhD thesis, California Institute of Technology, 1999.

[26] D. Jeon and M. Gharib. On circular cylinders undergoing two-degree-of-freedom forced motions. Journal of Fluids and Structures, 15(3-4):533-541, 2001.

[27] R. Govardhan and C.H.K. Williamson. Modes of vortex formation and frequency response of a freely vibrating cylinder. Journal of Fluid Mechanics, 420:85-130, 2000.

[28] F. Flemming and C.H.K. Williamson. Vortex-induced vibrations of a pivoted cylinder. Journal of Fluid Mechanics, 522:215-252, 2005.

[29] A. Sanchis, G. Saelevik, and J. Grue. Two-degree-of-freedom vortex-induced vibrations of a spring-mounted rigid cylinder with low mass ratio. Journal of Fluids and Structures, 24(6):907-919, 2008. 
[30] S. Kheirkhah, S. Yarusevych, and S. Narasimhan. Orbiting response in vortex-induced vibrations of a two degree-of-freedom pivoted circular cylinder. Journal of Fluids and Structures, 28:343-358, 2012.

[31] G.R. Franzini, R.T. Gonçalves, J.R Meneghini, and A.L.C. Fujarra. One and two degrees-of-freedom vortex-induced vibration experiments with yawed cylinders. Journal of Fluids and Structures, 42:401-420, 2013.

[32] N. Cagney and S. Balabani. Streamwise vortex-induced vibrations of cylinders with one and two degreesof-freedom. Journal of Fluid Mechanics, 758:702-727, 2014.

[33] S.-W. Kim, S.-J. Lee, C.-Y. Park, and D. Kang. An experimental study of a circular cylinder's two-degreeof-freedom motion induced by vortex. International Journal of Naval Architecture and Ocean Engineering, $8(4): 330-343,2016$.

[34] R.D. Blevins. Flow-induced vibration. 1990.

[35] K. Xu, Y. Ge, and D. Zhang. Wake oscillator model for assessment of vortex-induced vibration of flexible structures under wind action. Journal of Wind Engineering and Industrial Aerodynamics, 136:192-200, 2015. 